\title{
DAVID HILBERT AND HIS MATHEMATICAL WORK
}

\author{
HERMANN WEYL
}

A great master of mathematics passed away when David Hilbert died in Göttingen on February the 14th, 1943, at the age of eightyone. In retrospect it seems to us that the era of mathematics upon which he impressed the seal of his spirit and which is now sinking below the horizon achieved a more perfect balance than prevailed before and after, between the mastering of single concrete problems and the formation of general abstract concepts. Hilbert's own work contributed not a little to bringing about this happy equilibrium, and the direction in which we have since proceeded can in many instances be traced back to his impulses. No mathematician of equal stature has risen from our generation.

America owes him much. Many young mathematicians from this country, who later played a considerable role in the development of American mathematics, migrated to Göttingen between 1900 and 1914 to study under Hilbert. But the influence of his problems, his viewpoints, his methods, spread far beyond the circle of those who were directly inspired by his teaching.

Hilbert was singularly free from national and racial prejudices; in all public questions, be they political, social or spiritual, he stood forever on the side of freedom, frequently in isolated opposition against the compact majority of his environment. He kept his head clear and was not afraid to swim against the current, even amidst the violent passions aroused by the first world war that swept so many other scientists off their feet. It was not mere chance that when the Nazis "purged" the German universities in 1933 their hand fell most heavily on the Hilbert school and that Hilbert's most intimate collaborators left Germany either voluntarily or under the pressure of Nazi persecution. He himself was too old, and stayed behind; but the years after 1933 became for him years of ever deepening tragic loneliness.

It was another Germany in which he was born on January 23, 1862, and grew up. Königsberg, the eastern outpost of Prussia, the city of Kant, was his home town. Contrary to the habit of most German students who used to wander from university to university, Hilbert studied at home, and it was in his home university that he climbed the first rungs of the academic ladder, becoming Privatdozent and in due time ausserordentlicher Professor. During his entire life he preserved uncorrupted the characteristic Baltic accent. His reputa- 
tion as a leading algebraist was well established when on Felix Klein's initiative he was offered a full professorship at Göttingen in 1895 . From then on until the end of his life Hilbert remained in Göttingen. He was retired in 1930 .

When one inquires into the dominant influences acting upon Hilbert in his formative years one is puzzled by the peculiarly ambivalent character of his relationship to Kronecker: dependent on him, he rebels against him. Kronecker's work is undoubtedly of paramount importance for Hilbert in his algebraic period. But the old gentleman in Berlin, so it seemed to Hilbert, used his power and authority to stretch mathematics upon the Procrustean bed of arbitrary philosophical principles and to suppress such developments as did not conform: Kronecker insisted that existence theorems should be proved by explicit construction, in terms of integers, while Hilbert was an early champion of Georg Cantor's general set-theoretic ideas. Personal reasons added to the bitter feeling. ${ }^{1}$ A late echo of this old feud is the polemic against Brouwer's intuitionism with which the sexagenarian Hilbert opens his first article on "Neubegründung der Mathematik" (1922) : Hilbert's slashing blows are aimed at Kronecker's ghost whom he sees rising from his grave. But inescapable ambivalence even here -while he fights him he follows him: reasoning along strictly intuitionistic lines is found necessary by him to safeguard non-intuitionistic mathematics.

More decisive than any other influence for the young Hilbert at Königsberg was his friendship with Adolf Hurwitz and Minkowski. He got his thorough mathematical training less from lectures, teachers or books, than from conversation. "During innumerable walks, at times undertaken day after day," writes Hilbert in his obituary on Hurwitz, "we roamed in these eight years through all the corners of mathematical science, and Hurwitz with his extensive, firmly grounded and well ordered knowledge was for us always the leader." Closer and of a very intimate character was Hilbert's lifelong friendship with Minkowski. The Königsberg circle was broken up when Hurwitz in 1892 left for Zürich, soon to be followed by Minkowski. Hilbert first became Hurwitz's successor in Königsberg and then moved on to Göttingen. The year 1902 saw him and Minkowski reunited in Göttingen where a new chair of mathematics had been created for Minkowski. The two friends became the heroes of the great and brilliant period which our science experienced during the

${ }^{1}$ How Georg Cantor himself in his excitability suffered from Kronecker's opposition is shown by his violent outbursts in letters to Mittag-Leffler; see A. Schoenflies, Die Krisis in Cantors mathematischem Schaffen, Acta Math. vol. 50 (1928) pp. 1-23. 
following decade in Göttingen, unforgettable to those who lived through it. Klein, for whom mathematical research had ceased to be the central interest, ruled over it as a distant but benevolent god in the clouds. Too soon was this happy constellation dissolved by Minkowski's sudden death in 1909. In a memorial address before the Göttingen Gesellschaft der Wissenschaften, Hilbert spoke thus about his friend: "Our science, which we loved above everything, had brought us together. It appeared to us as a flowering garden. In this garden there are beaten paths where one may look around at leisure and enjoy oneself without effort, especially at the side of a congenial companion. But we also liked to seek out hidden trails and discovered many a novel view, beautiful to behold, so we thought, and when we pointed them out to one another our joy was perfect."

I quote these words not only as testimony of a friendship of rare depth and fecundity that was based on common scientific interest, but also because I seem to hear in them from afar the sweet flute of the Pied Piper that Hilbert was, seducing so many rats to follow him into the deep river of mathematics. If examples are wanted let me tell my own story. I came to Göttingen as a country lad of eighteen, having chosen that university mainly because the director of my high school happened to be a cousin of Hilbert's and had given me a letter of recommendation to him. In the fullness of my innocence and ignorance I made bold to take the course Hilbert had announced for that term, on the notion of number and the quadrature of the circle. Most of it went straight over my head. But the doors of a new world swung open for me, and I had not sat long at Hilbert's feet before the resolution formed itself in my young heart that I must by all means read and study whatever this man had written. And after the first year I went home with Hilbert's $Z$ ahlbericht under my arm, and during the summer vacation I worked my way through it-without any previous knowledge of elementary number theory or Galois theory. These were the happiest months of my life, whose shine, across years burdened with our common share of doubt and failure, still comforts my soul.

The impact of a scientist on his epoch is not directly proportional to the scientific weight of his research. To be sure, Hilbert's mathematical work is of great depth and universality, and yet his tremendous influence is not accounted for by it alone. Gauss and Riemann, to mention two other Göttingers, are certainly of no lesser stature than Hilbert, but they made little stir among their contemporaries and no "school" of devoted followers formed around them. No doubt this is due in part to the changing conditions of time, but the character of the men is probably more decisive. A taste for solitude, even 
obscurity, is in no way irreconcilable with great creative gifts. But Hilbert's was a nature filled with the zest of living, seeking intercourse with other people, above all with younger scientists, and delighting in the exchange of ideas. Just as he had learned from Hurwitz, so he taught his own pupils, at least those in whom he took a deeper personal interest: on far-flung walks through the woods surrounding Göttingen or, on rainy days, as "peripatetics" in his covered garden walk. His optimism, his spiritual passion, his unshakable faith in the supreme value of science, and his firm confidence in the power of reason to find simple and clear answers to simple and clear questions were irresistibly contagious. If Kant through critique and analysis arrived at the principle of the supremacy of practical reason, Hilbert incorporated, as it were, the supremacy of pure reason-sometimes with laughing arrogance (arrogancia in the Spanish sense), sometimes with the ingratiating smile of intellect's spoiled child, but most of the time with the seriousness of a man who believes and must believe in what is the essence of his own life. His enthusiasm was compatible with critical acumen, but not with scepticism. The snobbish attitude of pretended indifference, of "merely fooling around with things," or even of playful cynicism, were unknown in his circle. You had better think twice before you uttered a lie or an empty phrase to him: his directness could be something to be afraid of. He was enormously industrious and liked to quote Lichtenberg's saying: "Genius is industry." Yet for all this there was light and laughter around him. He had great suggestive power; it sometimes lifted even mediocre minds high above their natural level to astonishing, though isolated achievements. I do not remember which mathematician once said to him: "You have forced us all to consider important those problems which you considered important." His vision and experience inspired confidence in the fruitfulness of the hints he dropped. He did not hide his light under a bushel. In his papers one encounters not infrequently utterances of pride in a beautiful or unexpected result, and in his legitimate satisfaction he sometimes did not give to his predecessors on whose ideas he built all the credit they deserved. The problems of mathematics are not isolated problems in a vacuum; there pulses in them the life of ideas which realize themselves in concreto through our human endeavors in our historical existence, but forming an indissoluble whole transcend any particular science. Hilbert had the power to evoke this life; against it he measured his individual scientific efforts and felt responsible for it in his own sphere. In this sense he was a philosopher, not in the sense of adhering to one of the established epistemological or metaphysical doctrines. Does not in 
such personal qualities of the academic teacher, rather than in any objectivities or universally accepted metaphysics, lie the answer to Hutchins's quest for a true universitas literarum?

Were it my aim to give a full picture of Hilbert's personality I should have to touch upon his attitude regarding the great powers in the lives of men: social and political organization, art, religion, morals and manners, family, friendship, love, and I should also probably have to indicate some of the shadows cast by so much light. I wanted merely to sketch the mathematical side of his personality in an attempt to explain, however incompletely, the peculiar charm and the enormous influence which he exerted. In appraising the latter one must not overlook the environmental factor. A German university in a small town like Göttingen, especially in the halcyon days before 1914, was a favorable milieu for the development of a scientific school. The high social prestige of the professors and everything connected with the university created an atmosphere the like of which has hardly ever existed in America. And once a band of disciples had gathered around Hilbert, intent on research and little worried by the chore of teaching, how could they fail to stimulate one another! We have seen the same thing happening here at Princeton during the first years of the Institute for Advanced Study; there is a kind of snowball effect in the formation of such condensation points of scientific research.

Before giving a more detailed account of Hilbert's work, it remains to characterize in a few words the peculiarly Hilbertian brand of mathematical thinking. It is reflected in his literary style which is one of great lucidity. It is as if you were on a swift walk through a sunny open landscape; you look freely around, demarcation lines and connecting roads are pointed out to you, before you must brace yourself to climb the hill; then the path goes straight up, no ambling around, no detours. His style has not the terseness of many of our modern authors in mathematics, which is based on the assumption that printer's labor and paper are costly but the reader's effort and time are not. In carrying out a complete induction Hilbert finds time to develop the first two steps before formulating the general conclusion from $n$ to $n+1$. How many examples illustrate the fundamental theorems of his algebraic papers-examples not constructed ad hoc, but genuine ones worth being studied for their own sake!

In Hilbert's approach to mathematics, simplicity and rigor go hand in hand. The generation before him, nay even most analysts of his time, felt the growing demand for rigor imposed upon analysis by the critique of the 19th century, which culminated in Weierstrass, as a 
heavy yoke that made their steps dragging and awkward. Hilbert did much to change that attitude. In his famous address, Mathematische Probleme, delivered before the Paris Congress in 1900, he stresses the importance of great concrete fruitful problems. "As long as a branch of science," says he, "affords an abundance of problems, it is full of life; want of problems means death or cessation of independent development. Just as every human enterprise prosecutes final aims, so mathematical research needs problems. Their solution steels the force of the investigator; thus he discovers new methods and viewpoints and widens his horizon." "One who without a definite problem before his eyes searches for methods, will probably search in vain." The methodical unity of mathematics was for him a matter of belief and experience. Again I quote his own words: "The question is forced upon us whether mathematics is once to face what other sciences long ago experienced, namely the falling apart into subdivisions whose representatives are hardly able to understand each other and whose connections for this reason will become ever looser. I neither wish nor believe it. The science of mathematics as I see it is an indivisible whole, an organism whose ability to survive rests on the connection between its parts." A characteristic feature of Hilbert's method is a peculiarly direct attack on problems, unfettered by algorithms; he always goes back to the questions in their original simplicity. An outstanding example is his salvage of Dirichlet's principle which had fallen a victim of Weierstrass's criticism, but his work abounds in similar examples. His strength, equally disdainful of the convulsion of Herculean efforts and of surprising tricks and ruses, is combined with an uncompromising purity.

Hilbert helped the reviewer of his work greatly by seeing to it that it is rather neatly cut into different periods during each of which he was almost exclusively occupied with one particular set of problems. If he was engrossed in integral equations, integral equations seemed everything; dropping a subject, he dropped it for good and turned to something else. It was in this characteristic way that he achieved universality. I discern five main periods: $i$. Theory of invariants (1885-1893). ii. Theory of algebraic number fields (18931898). iii. Foundations, (a) of geometry (1898-1902), (b) of mathematics in general (1922-1930). iv. Integral equations (1902-1912). v. Physics (1910-1922). The headings are a little more specific than they ought to be. Not all of Hilbert's algebraic achievements are directly related to invariants. His papers on calculus of variations are here lumped together with those on integral equations. And of course there are some overlappings and a few stray children who break 
the rules of time, the most astonishing his proof of Waring's theorem in 1909.

His Paris address on "Mathematical problems" quoted above straddles all fields of our science. Trying to unveil what the future would hold in store for us, he posed and discussed twenty-three unsolved problems which have indeed, as we can now state in retrospect, played an important role during the following forty odd years. A mathematician who had solved one of them thereby passed on to the honors class of the mathematical community.

\section{LITERATURE}

Hilbert's Gesammelte Abhandlungen were published in 3 volumes by J. Springer, Berlin, 1932-35. This edition contains his Zahlbericht, but not his two books:

Grundlagen der Geometrie, 7th ed., Leipzig, 1930.

Grundzilge einer allgemeinen Theorie der linearen Integralgleichungen, Leipzig and Berlin, 1912.

Hilbert is co-author of the following works:

R. Courant and D. Hilbert, Methoden der mathematischen Physik, Berlin, vol. 1, 2d ed., 1931, vol. 2, 1937.

D. Hilbert and W. Ackermann, Grundzilge der theoretischen Logik, Berlin, 1928.

D. Hilbert and S. Cohn-Vossen, Anschauliche Geometrie, Berlin, 1932. 1939.

D. Hilbert and P. Bernays, Grundlagen der Mathematik, Berlin, vol. 1, 1934, vol. 2,

The Collected Papers contain articles by B. L. van der Waerden, H. Hasse, A. Schmidt, P. Bernays, and E. Hellinger, on Hilbert's work in algebra, in number theory, on the foundations of geometry and arithmetics, and on integral equations. These articles trace the development after Hilbert, giving ample references. The reader may also consult a number of Die Naturwissenschaften vol. 10 (1922) pp. 65-104, dedicated to Hilbert, which surveys his work prior to 1922, and an article by L. Bieberbach, Ueber den Einfluss von Hilberts Pariser Vortrag uber "Mathematische Probleme" auf die Entwicklung der Mathematik in den letzten dreissig Jahren, Die Naturwissenschaften vol. 18 (1930) pp. 1101-1111. O. Blumenthal wrote a life of Hilbert (Collected Papers, vol. 3, pp. 388-429).

I omit all quotations of literature covered by these articles.

\section{THEORY OF INVARIANTS}

The classical theory of invariants deals with polynomials $J$ $=J\left(x_{1}, \ldots x_{n}\right)$ depending on the coefficients $x_{1}, \ldots, x_{n}$ of one or several ground forms of a given number of arguments $\eta_{1}, \cdots, \eta_{0}$. Any linear substitution $s$ of determinant 1 of the $g$ arguments induces a certain linear transformation $U(s)$ of the variable coefficients 
$x_{1}, \cdots, x_{n}, x \rightarrow x^{\prime}=U(s) x$, whereby $J=J\left(x_{1} \cdots x_{n}\right)$ changes into a new form $J\left(x_{1}^{\prime} \cdots x_{n}^{\prime}\right)=J^{*}\left(x_{1} \cdots x_{n}\right) . J$ is an invariant if $J^{\circ}=J$ for every $s$. (The restriction to unimodular transformations $s$ enables one to avoid the more involved concept of relative invariants and to remove the restriction to homogeneous polynomials, with the convenient consequence that the invariants form a ring.) The classical problem is a special case of the general problem of invariants in which $s$ ranges over an arbitrary given abstract group $\Gamma$ and $s \rightarrow U(s)$ is any representation of that group (that is, a law according to which every element $s$ of $\Gamma$ induces a linear transformation $U(s)$ of the $n$ variables $x_{1}, \cdots, x_{n}$ such that the composition of group elements is reflected in composition of the induced transformations). The development before Hilbert had led up to two main theorems, which however had been proved in very special cases only. The first states that the invariants have a finite integrity basis, or that we can pick a finite number among them, say $i_{1}, \cdots, i_{m}$, such that every invariant $J$ is expressible as a polynomial in $i_{1}, \cdots, i_{m}$. An identical relation between the basic invariants $i_{1}, \cdots, i_{m}$ is a polynomial $F\left(z_{1} \cdots z_{m}\right)$ of $m$ independent variables $z_{1}, \cdots, z_{m}$ which vanishes identically by virtue of the substitution

$$
z_{1}=i_{1}\left(x_{1} \cdots x_{n}\right), \cdots, z_{m}=i_{m}\left(x_{1} \cdots x_{n}\right) .
$$

The second main theorem asserts that the relations have a finite ideal basis, or that one can pick a finite number among them, say $F_{1}, \cdots, F_{h}$, such that every relation $F$ is expressible in the form

$$
F=Q_{1} F_{1}+\cdots+Q_{h} F_{h},
$$

the $Q_{i}$ being polynomials of the variables $z_{1}, \cdots, z_{m}$.

I venture the guess that Hilbert first succeeded in proving the second theorem. The relations $F$ form a subset within the ring $k\left[z_{1} \cdots z_{m}\right]$ of all polynomials of $z_{1}, \cdots, z_{m}$ the coefficients of which lie in a given field $k$. When Hilbert found his simple proof he could not fail to notice that it applied to any set of polynomials $\Sigma$ whatsoever and he thus discovered one of the most fundamental theorems of algebra, which was instrumental in ushering in our modern abstract approach, namely:

(A) Every subset $\Sigma$ of the polynomial ring $k\left[z_{1} \cdots z_{m}\right]$ has a finite ideal basis.

Is it bad metaphysics to add that his proof turned out so simple because the proposition holds in this generality? The proof proceeds by the adjoining of one variable $z_{i}$ after the other, the individual step 
being taken care of by the statement: If a given ring $r$ satisfies the condition $(\mathrm{P})$ : that every subset of $r$ has a finite ideal basis, then the ring $r[z]$ of polynomials of a single variable $z$ with coefficients in $r$ satisfies the same condition (P). Once this is established one gets not only (A) but also an arithmetic refinement discussed by Hilbert in which the field $k$ of rational numbers is replaced by the ring of rational integers.

The subset $\Sigma$ of relations to which Hilbert applies his theorem (A) is itself an ideal, and thus the ideal $\left\{F_{1}, \cdots, F_{h}\right\}$, that is, the totality of all elements of the form (1), $Q_{i} \varepsilon k\left[z_{1} \cdots z_{m}\right]$, not only contains, but coincides with, $\Sigma$. The proof, however, works even if $\Sigma$ is not an ideal, and yields at one stroke (1) the enveloping ideal $\{\Sigma\}$ of $\Sigma$ and (2) the reduction of that ideal to a finite basis, $\{\Sigma\}=\left\{F_{1}, \cdots, F_{h}\right\}$.

Construction of a full set of relations $F_{1}, \cdots, F_{h}$ would finish the investigation of the algebraic structure of the ring of invariants were it true that any relation $F$ can be represented in the form (1) in one way only. But since, generally speaking, this is not so, we must ask for the "vectors of polynomials" $M=\left(M_{1}, \cdots, M_{h}\right)$ for which $M_{1} F_{1}+\cdots+M_{h} F_{h}$ vanishes identically in $z$ (syzygy of first order). These linear relations $M$ between $F_{1}, \cdots, F_{h}$ again form an ideal to which Theorem (A) is applicable, the basis of the $M$ thus obtained giving rise to syzygies of the second order. To the first two main theorems Hilbert adds a third to the effect that if redundance is avoided, the chain of syzygies breaks off after at most $m$ steps.

All this hangs in the air unless we can establish the first main theorem, which is of an altogether different character because it asks for an integrity, not an ideal basis. Discussing invariants we operate in the ring $k_{x}=k\left[x_{1} \cdots x_{n}\right]$ of polynomials of $x_{1}, \cdots, x_{n}$ in a given field $k$. Hilbert applies his Theorem (A) to the totality $J$ of all invariants $J$ for which $J(0, \cdots, 0)=0$ (a subring of $k_{x}$ which, by the way, is not an ideal!) and thus determines' an ideal basis $i_{1}, \cdots, i_{m}$ of $J$. Each of the invariants $i=i_{r}$ may be decomposed into a sum $i=i^{(1)}+i^{(2)}+\cdots$ of homogeneous forms of degree $1,2, \cdots$, and as the summands are themselves invariants, the $i_{r}$ may be assumed to be homogeneous forms of degrees $\nu_{r} \geqq 1$. Hilbert then claims that the $i_{1}, \cdots, i_{m}$ constitute an integrity basis for all invariants. I use a finite group $\Gamma$ consisting of $N$ elements $s$ (although this case of the general problem of invariants was never envisaged by Hilbert himself) in order to illustrate the idea by which the transition is made. Every invariant $J$ is representable in the form

$$
J=c+L_{1} i_{1}+\cdots+L_{m} i_{m}
$$


where $c$ is the constant $J(0)$. If $J$ is of degree $\nu$ one may lop off in $L_{r}$ all terms of higher degree than $\nu-\nu_{r}$ without destroying the equation. If it were possible by some process to change the coefficients $L$ in (2) into invariants, the desired result would follow by induction with respect to the degree of $J$. In the case of a finite group such a process is readily found: the process of averaging. The linear transformation $U(s)$ of the variables $x_{1}, \cdots, x_{n}$ induced by $s$ carries (2) into

$$
J=c+L_{1}^{s} \cdot i_{1}+\cdots+L_{m}^{s} \cdot i_{m} \text {. }
$$

Summation over $s$ and subsequent division by the number $N$ yields the relation

$$
J=c+L_{1}^{*} i_{1}+\cdots+L_{m}^{*} i_{m}
$$

where

$$
L_{r}^{*}=\frac{1}{N} \cdot \sum_{s} L_{r}^{*} .
$$

It is of the same nature as (2), except for the decisive fact that according to their formation the new coefficients $L^{*}$ are invariants. ${ }^{2}$

Actually Hilbert had to do, not with a finite group but with the classical problem in which the group $\Gamma$ consists of all linear transformations $s$ of $g$ variables $\eta_{1}, \cdots, \eta_{g}$, and instead of the averaging process he had to resort to a differentiation process invented by Cayley, the so-called Cayley $\Omega$-process, which he skillfully adapted for his end. (It is essential in Cayley's process that the $g^{2}$ components of the matrix $s$ are independent variables; instead of the absolutely invariant polynomials $J$ one has to consider relatively invariant homogeneous forms each of which has a definite degree and weight.)

Hilbert's theorem (A) is the foundation stone of the general theory of algebraic manifolds. Let us now think of $k$ more specifically as the field of all complex numbers. It seems natural to define an algebraic manifold in the space of $n$ coordinates $x_{1}, \cdots, x_{n}$ by a number of simultaneous algebraic equations $f_{1}=0, \cdots, f_{h}=0\left(f_{i} \varepsilon k_{x}\right)$. According to Theorem (A), nothing would be gained by admitting an infinite number of equations. Let us denote by $Z\left(f_{1}, \cdots, f_{h}\right)$ the set of points $x=\left(x_{1}, \cdots, x_{n}\right)$ where $f_{1}, \cdots, f_{h}$ and hence all elements of the idear $\mathcal{F}=\left\{f_{1}, \cdots, f_{h}\right\}$ vanish simultaneously. $g$ vanishes on $Z\left(f_{1}, \cdots, f_{h}\right)$ whenever $g \varepsilon\left\{f_{1}, \cdots, f_{h}\right\}$, but the converse is not generally true. For

2 The example of finite groups is used here as an illustration only. Indeed, a direct elementary proof of the first main theorem for finite groups that makes no use of Hilbert's principle (A) has been given by E. Noether, Math. Ann. vol. 77 (1916) p. 89. In dividing by $N$ we have assumed the field $k$ to be of characteristic zero. 
instance $x_{1}$ vanishes wherever $x_{1}^{8}$ does, and yet $x_{1}$ is not of the form $x_{1}^{3} \cdot q\left(x_{1} \cdots x_{n}\right)$. The language of the algebraic geometers distinguishes here between the simple plane $x_{1}=0$ and the triple plane, although the point set is the same in both cases. Hence what they actually mean by an algebraic manifold is the polynomial ideal and not the point set of its zeros. But even if one cannot expect that every polynomial $g$ vanishing on $Z\left(f_{1}, \cdots, f_{h}\right)=Z(\mathcal{F})$ is contained in the ideal $\mathcal{F}=\left\{f_{1}, \cdots, f_{h}\right\}$ one hopes that at least some power of $g$ will be. Hilbert's "Nullstellensatz" states that this is true, at least if $k$ is the field of complex numbers. It holds in an arbitrary coefficient field $k$ provided one admits points $x$ the coordinates $x_{i}$ of which are taken from $k$ or any algebraic extension of $k$. Clearly this Nullstellensatz goes to the root of the very concept of algebraic manifolds. ${ }^{3}$

Actually Hilbert conceived it as a tool for the investigation of invariants. As we are now dealing with the full linear group let us consider homogeneous invariants only and drop the adjective homogeneous. Exclude the constants (the invariants of degree 0). Suppose we have ascertained $\mu$ non-constant invariants $J_{1}, \ldots, J_{\mu}$ such that every non-constant invariant vanishes wherever they vanish simultaneously. An ideal basis of the set $\exists$ of all non-constant invariants certainly meets the demand, but a system $J_{1}, \ldots, J_{\mu}$ may be had much more cheaply. Indeed, by a beautiful combination of ideas Hilbert proves that if for a given point $x=x^{0}$ there exists at all an invariant which neither vanishes for $x=x^{0}$ nor reduces to a constant, then there exists such an invariant whose weight does not exceed a certain a priori limit $W$ (for example, $W=9 n(3 n+1)^{8}$ for a ternary ground form of order $n$ ). Hence the $J_{1}, \cdots, J_{\mu}$ may be chosen from the invariants of weight not greater than $W$, and they thus come within the grasp of explicit algebraic construction.

When Hilbert published his proof for the existence of a finite ideal basis, Gordan the formalist, at that time looked upon as the king of invariants, cried out: "This is not mathematics, it is theology!" Hilbert remonstrated then, as he did all his life, against the disparagement of existential arguments as "theology," but we see how, by digging deeper, he was able to meet Gordan's constructive demands. By combining the Nullstellensatz with the Cayley process he further showed that every invariant $J$ is an integral algebraic (though not an integral rational) function of $J_{1}, \cdots, J_{\mu}$, satisfying an equation

${ }^{3}$ B. L. van der Waerden's book Moderne Algebra, vol. 2, 2nd ed., 1940, gives on pp. 1-72 an excellent account of the general algebraic concepts and facts with which we are here concerned. 


$$
J^{e}+G_{1} J^{e-1}+\cdots+G_{0}=0
$$

in which the $G$ 's are polynomials of $J_{1}, \cdots, J_{\mu}$. Hence it must be possible by suitable algebraic extensions to pass from $J_{1}, \cdots, J_{\mu}$ to a full integrity basis. From there on familiar algebraic patterns such as were developed by Kronecker and as are amenable to explicit construction may be followed.

After the formal investigations from Cayley and Sylvester to Gordan, Hilbert inaugurated a new epoch in the theory of invariants. Indeed, by discovering new ideas and introducing new powerful methods he not only brought the subject up to the new level set for algebra by Kronecker and Dedekind, but made such a thorough job of it that he all but finished it, at least as far as the full linear group is concerned. With justifiable pride he concludes his paper, Ueber die vollen Invariantensysteme, with the words: "Thus I believe the most important goals of the theory of the function fields generated by invariants have been attained," and therewith quits the scene. ${ }^{4}$

Of later developments which took place after Hilbert quit, two main lines seem to me the most important: (1) The averaging process, which we applied above to finite groups, carries over to continuous compact groups. By this transcendental process of integration over the group manifold, Adolf Hurwitz treated the real orthogonal group. The method has been of great fertility. The simple remark that invariants for the real orthogonal group are eo ipso also invariant under the full complex orthogonal group indicates how the results can be transferred even to non-compact groups, in particular, as it turns out, to all semi-simple Lie groups. (2) Today the theory of invariants for arbitrary groups has taken its natural place within the frame of the theory of representations of groups by linear substitutions, a development which owes its greatest impulse to G. Frobenius.

Although the first main theorem has been proved for wide classes of groups $\Gamma$ we do not yet know whether it holds for every group. Such attempts as have been made to establish it in this generality were soon discovered to have failed. A promising line for an algebraic attack is outlined in item 14 of Hilbert's Paris list of Mathematical Problems.

Having dwelt in such detail on Hilbert's theory of invariants, we must be brief with regard to his other, more isolated, contributions to algebra. The first paper in which the young algebraist showed his real

\footnotetext{
"I recommend to the reader's attention a brief resumé of his invariant-theoretic work which Hilbert himself wrote for the International Mathematical Congress held at Chicago in conjunction with the World Fair in 1893; Collected Papers, vol. 2, item 23.
} 
mettle concerns the conditions under which a form with real coeffcients is representable as a sum of squares of such forms, in particular with the question whether the obviously necessary condition that the form be positive for all real values of its arguments is sufficient. By ingenious continuity arguments and algebraic constructions Hilbert finds three special cases for which the answer is affirmative, among them of course the positive definite quadratic forms, but counterexamples for all other cases. Similar methods recur in two papers dealing with the attractive problem of the maximum number of real ovals of an algebraic curve or surface and their mutual position. Hilbert conjectured that, irrespective of the number of variables, every rational function with real (or rational) coefficients is a sum of squares of such functions provided its values are positive for real values of the arguments; and in his Grundlagen der Geometrie he pointed out the role of this fact for the geometric constructions with ruler and "Eichmass." Later O. Veblen conceived, as the basis of the distinction between positive and negative in any field, the axiom that no square sum equals zero. Independently of him, E. Artin and O. Schreier developed a detailed theory of such "real fields," and by means of it Artin succeeded in proving Hilbert's conjecture. ${ }^{5}$

In passing I mention Hilbert's irreducibility theorem according to which one may substitute in an irreducible polynomial suitable integers for all of the variables but one without destroying the irreducibility of the polynomial, and his paper on the solution of the equation of ninth degree by functions with a minimum number of arguments. They became points of departure for much recent algebraic work (E. Noether, N. Tschebotareff and others). Finally, it ought to be recorded that on the foundations laid by Hilbert a detailed theory of polynomial ideals was erected by E. Lasker and F. S. Macaulay which in turn gave rise to Emmy Noether's general axiomatic theory of ideals. Thus in the field of algebra, as in all other fields, Hilbert's conceptions proved of great consequence for the further development.

\section{Algebraic NUMBER FIELDS}

When Hilbert, after finishing off the invariants, turned to the theory of algebraic number fields, the ground had been laid by Dirichlet's analysis of the group of units more than forty years before, and by Kummer's, Dedekind's and Kronecker's introduction of ideal divisors. The theory deals with an algebraic field $\kappa$ over the field $k$

5 O. Veblen, Trans. Amer. Math. Soc. vol. 7 (1906) pp. 197-199. E. Artin and O. Schreier, Abh. Math. Sem. Hamburgischen Univ. vol. 5 (1926) pp. 85-99; E. Artin, ibid. pp. 100-115. 
of rational numbers. One of the most important general results beyond the foundations had been discovered by Dedekind, who showed that the rational prime divisors of the discriminant of $\kappa$ are at the same time those primes whose ideal prime factors in $\kappa$ are not all distinct (ramified primes). $l$ being a rational prime, the adjunction to $\kappa$ of the $l$ th root of a number $\alpha$ in $\kappa$ yields a relative cyclic field $K=\kappa\left(\alpha^{1 / l}\right)$ of degree $l$ over $\kappa$, provided $\kappa$ contains the lth root of unity $\zeta=e^{2 \pi i / l}$ (and according to Lagrange, the most general relative cyclic field of degree $l$ over $\kappa$ is obtained in this fashion). It may be said that it was this circumstance which forced Kummer, as he tried to prove Fermat's theorem of the impossibility of the equation $\alpha^{l}+\beta^{l}=\gamma^{l}$, to pass from the rational ground field $k$ to the cyclotomic field $\kappa_{l}=k(\zeta)$ and then to conceive his ideal numbers in $\kappa_{l}$ and to investigate whether the number of classes of equivalent ideal numbers in $\kappa_{l}$ is prime to $l$. Hilbert sharpened his tools in resuming Kummer's study of the relative cyclic fields of degree $l$ over $\kappa_{l}$, which he christened "Kummer fields."

His own first important contribution was a theory of relative Galois fields $K$ over a given algebraic number field $\kappa$. His main concern is the relation of the Galois group $\Gamma$ of $K / \kappa$ to the way in which the prime ideals of $\kappa$ decompose in $K$. Given a prime ideal $\mathfrak{B}$ in $K$ of relative degree $f$, those substitutions $s$ of $\Gamma$ for which $s \mathfrak{B}=\mathfrak{B}$ form the splitting group. As always in Galois theory one constructs the corresponding subfield of $K / \kappa$ (splitting field), to which a number of $K$ belongs if it is invariant under all substitutions of the splitting group. The substitutions $t$ which carry every integer $\mathrm{A}$ in $K$ into one, $t \mathbf{A}$, that is congruent to $A$ mod $\mathfrak{P}$ form an invariant subgroup of the splitting group of index $f$, called the inertial group, and the corresponding field (inertial field) is sandwiched in between the splitting field and $K$. Let $\mathfrak{p}$ be the prime ideal in $\kappa$ into which $\mathfrak{P}$ goes, and $\mathfrak{P}^{e}$ the exact power of $\mathfrak{B}$ by which $\mathfrak{p}$ is divisible. $I$ indicate the nature of Hilbert's results by the following central theorem of his: In the splitting field of $\mathfrak{B}$ the prime ideal $\mathfrak{p}$ in $\kappa$ splits off the prime factor $\mathfrak{p}^{*}=\mathfrak{B}^{\boldsymbol{e}}$ of degree 1 (therefore the name!); in passing from the splitting to the inertial field $\mathfrak{p}^{*}$ stays prime but its degree increases to $f$; in passing from the inertial to the full field $K, \mathfrak{p}^{*}$ breaks up into $e$ equal prime factors $\mathfrak{B}$ of the same degree $f$. For later application I add the following remarks. If $\mathfrak{P}$ goes into $\mathfrak{p}$ in the first power only, $e=1$ (which is necessarily so provided $p$ is not a divisor of the relative discriminant of $K / \kappa)$, then the inertial group consists of the identity only. In that case the theory of Galois's strictly finite fields shows that the splitting group is cyclic of order $f$ and that its elements $1, s, s^{2}, \cdots, s^{f-1}$ are 
uniquely determined by the congruences

$$
s \mathrm{~A} \equiv \mathrm{A}^{P}, \quad s^{2} \mathrm{~A} \equiv \mathrm{A}^{P^{2}}, \cdots
$$

holding for every integer A. Here $P$ is the number of residues in $\kappa$ modulo $p$ and thus $P^{f}$ the number of residues in $K$ modulo $\mathfrak{B}$. Today we call $s=\sigma(\mathfrak{B})$ the Frobenius substitution of $\mathfrak{B}$; it is of paramount importance that one particular generating substitution of the splitting group may thus be distinguished among all others. One readily sees that for any substitution $u$ of the Galois group $\sigma(u \mathfrak{B})=u^{-1} \cdot \sigma(\mathfrak{B}) \cdot u$. Thus if the Galois field $K / \kappa$ is Abelian, the substitution $\sigma(\mathfrak{B})=\sigma(u \mathfrak{B})$ depends on $\mathfrak{p}$ only and may be denoted by $\left(\frac{K}{p}\right)$.

In 1893 the Deutsche Mathematiker-Vereinigung asked Hilbert and Minkowski to submit within two years a report on number theory. Minkowski dropped out after a while. Hilbert's monumental report Die Theorie der algebraischen Zahlkörper appeared in the Jahresberichte of 1896 (the preface is dated April 1897). What Hilbert accomplished is infinitely more than the Vereinigung could have expected. Indeed, his report is a jewel of mathematical literature. Even today, after almost fifty years, a study of this book is indispensable for anybody who wishes to master the theory of algebraic numbers. Filling the gaps by a number of original investigations, Hilbert welded the theory into an imposing unified body. The proofs of all known theorems he weighed carefully before he decided in favor of those "the principles of which are capable of generalization and the most useful for further research." But before such a selection could be made that "further research" had to be carried out! Meticulous care was given to the notations, with the result that they have been universally adopted (including, to the American printer's dismay, the German letters for ideals!) He greatly simplified Kummer's theory, which rested on very complicated calculations, and he introduced those concepts and proved a number of those theorems in which we see today the foundations of a general theory of relative Abelian fields. The most important concept is the norm residue symbol, a pivotal theorem on relative cyclic fields, his famous Satz 90 (Collected Works, vol. I, p. 149). From the preface in which he describes the general character of number theory, and the topics covered by his report in particular, let me quote one paragraph:

"The theory of number fields is an edifice of rare beauty and harmony. The most richly executed part of this building, as it appears to me, is the theory of Abelian fields which Kummer by his work on the higher laws of reciprocity, and Kronecker by his investigations 
on the complex multiplication of elliptic functions, have opened up to us. The deep glimpses into the theory which the work of these two mathematicians affords reveals at the same time that there still lies an abundance of priceless treasures hidden in this domain, beckoning as a rich reward to the explorer who knows the value of such treasures and with love pursues the art to win them."

Hilbert himself was the miner who during the following two years brought to light much of the hidden ore. The analogy with the corresponding problems in the realm of algebraic functions of one variable where Riemann's powerful instruments of topology and Abelian integrals are available was for him a guiding principle throughout (cf. his remarks in item 12 of his Paris Problems). It is a great pleasure to watch how, step by step, advancing from the special to the general, Hilbert evolves the adequate concepts and methods, and the essential conclusions emerge. I mention his great paper dealing with the relative quadratic fields, and his last and most important Ueber die Theorie der relativ Abelschen Zahlkörper. On the basis of the examples he carried through in detail, he conceived as by divination and formulated the basic facts about the so-called class fields. Whereas Hilbert's work on invariants was an end, his work on algebraic numbers was a beginning. Most of the labor of such number theorists of the last decades, as Furtwängler, Takagi, Hasse, Artin, Chevalley, has been devoted to proving the results anticipated by Hilbert. By deriving from the $\zeta$-function the existence of certain auxiliary prime ideals, Hilbert had leaned heavily on transcendental arguments. The subsequent development has gradually eliminated these transcendental methods and shown that though they are the fitting and powerful tool for the investigation of the distribution of prime ideals they are alien to the problem of class fields. In attempting to describe the main issues I shall not ignore the progress and simplification due to this later development.

Hilbert's theory of norm residues is based on the following discoveries of his own: (1) he conceived the basic idea and defined the norm residue symbol for all non-exceptional prime spots; (2) he realized the necessity of introducing infinite prime spots; (3) he formulated the general law of reciprocity in terms of the norm symbol; (4) he saw that by means of that law one can extend the definition of the norm symbol to the exceptional prime spots where the really interesting things happen.- It was an essential progress when E. Artin later (5) replaced the roots of unity by the elements of the Galois group as values of the residue symbol. In sketching Hilbert's problems I shall make use of this idea of Artin's and also of the abbreviating language 
of (6) Hensel's $p$-adic numbers and (7) Chevalley's ideles. ${ }^{6}$

As everybody knows an integer $a$ indivisible by the prime $p \neq 2$ is said to be a quadratic residue if the congruence $x^{2} \equiv a(\bmod p)$ is solvable. Gauss introduced the symbol $\left(\frac{a}{p}\right)$ which has the value +1 or -1 according to whether $a$ is a quadratic residue or non-residue $\bmod p$, and observed that it is a character, $\left(\frac{a}{p}\right) \cdot\left(\frac{a^{\prime}}{p}\right)=\left(\frac{a a^{\prime}}{p}\right)$. Indeed, the $p$ residues modulo $p$-as whose representatives one may take $0,1, \cdots$, $(p-1)$-form a field, and after exclusion of 0 a group in which the quadratic residues form a subgroup of index 2. Let $K=k\left(b^{1 / 2}\right)$ be a quadratic field which arises from the rational ground field $k$ by adjunction of the square root of the rational number $b$. An integer $a \neq 0$ is called by Hilbert $a p$-adic norm in $K$ if modulo any given power of $p$ it is congruent to the norm of a suitable integer in $K$. He sets $\left(\frac{a, K}{p}\right)=+1$ if $a$ is $p$-adic norm, -1 in the opposite case, and finds that this $p$-adic norm symbol again is a character. The investigation of numbers modulo arbitrarily high powers of $p$ was systematized by $K$. Hensel in the form of his $p$-adic numbers, and I repeat Hilbert's definition in this language: "The rational number $a \neq 0$, or more generally the $p$-adic number $a_{p} \neq 0$, is a $p$-adic norm in $K$ if the equation

$$
a_{p}=\mathrm{Nm}\left(x+y b^{1 / 2}\right)=x^{2}-b y^{2}
$$

has a $p$-adic solution $x=x_{p}, y=y_{p}$; the norm symbol $\left(a_{p}, K\right)$ equals +1 or -1 according to whether or not $a_{p}$ is (p-adic) norm in $K$." The $p$-adic numbers form a field $k(p)$ and after exclusion of 0 a multiplicative group $G_{p}$ in which, according to Hilbert's result, the $p$-adic norms in $K$ form a subgroup of index 2 or 1 . The cyclic nature of the factor group is the salient point. One easily finds that the $p$-adic squares form a subgroup $G_{p}^{2}$ of index 4 if $p \neq 2$, of index 8 if $p=2$, and thus the factor group $G_{p} / G_{p}^{2}$ is not cyclic and could not be described by a single character. Of course every $p$-adic square is a $p$-adic norm in $K$. Both steps, the substitution of $K$-norms for squares and the passage from the modulus $p$ to arbitrarily high powers of $p$; the first step amounting to a relaxation, the second to a sharpening of Gauss's condition for quadratic residues; are equally significant for the success of Hilbert's definition.

Every $p$-adic number $a_{p} \neq 0$ is of the form $p^{h} \cdot e_{p}$ where $e_{p}$ is a $p$-adic unit, and thus $a_{p}$ is of a definite order $h$ (at $\left.p\right)$. An ordinary rational number $a$ coincides with a definite $p$-adic number $I_{p}(a)=a_{p}$. Here $I_{p}$ symbolizes a homomorphic projection of $k$ into $k(p)$ :

$$
I_{p}\left(a+a^{\prime}\right)=I_{p}(a)+I_{p}\left(a^{\prime}\right), \quad I_{p}\left(a a^{\prime}\right)=I_{p}(a) \cdot I_{p}\left(a^{\prime}\right) .
$$

${ }^{6}$ The latest account of the theory is C. Chevalley's paper La theorie $d u$ corps de classes, Ann. of Math. vol. 41 (1940) pp. 394-418. 
The character $\left(\frac{a, K}{p}\right)$ is identical with $\left(I_{p}(a), K\right)$.

We come to Hilbert's second discovery: he realized that simple laws will not result unless one adds to the "finite prime spots" $p$ one infinite prime spot $q$. By definition the $q$-adic numbers are the real numbers and $I_{q}(a)$ is the real number with which the rational number $a$ coincides. Hence the real number $a_{q}$ is a $q$-adic norm in $K$ if the equation $a_{q}=x^{2}-b y^{2}$ has a solution in real numbers $x, y$. Clearly if $b>0$ or $K$ is real, this is the case for every $a_{q}$; if however $b<0$ or $K$ is imaginary, only the positive numbers $a_{q}$ are $q$-adic norms. Hence

$$
\left(a_{q}, K\right)=1 \text { if } K \text { real; } \quad\left(a_{q}, K\right)=\operatorname{sgn} a_{q} \text { if } K \text { imaginary. }
$$

The fact that the norm symbol is a character is thus much more easily verified for the infinite prime spot than for the finite ones.

Hilbert's third observation is to the effect that Gauss's reciprocity law with its two supplements may be condensed into the elegant formula

$$
\prod_{p}\left(I_{p}(a), K\right)=\prod_{p}\left(\frac{a, K}{p}\right)=1,
$$

the product extending over the infinite and all finite prime spots $p$. There is no difficulty in forming this product because almost all factors (that is, all factors with but a finite number of exceptions) equal unity. Indeed, if the prime $p$ does not go into the discriminant of $K$, then $\left(a_{p}, K\right)=1$ for every $p$-adic unit $a_{p}$. Formula (3) is the first real vindication for the norm residue idea, which must have given Hilbert the assurance that the higher reciprocity laws had to be formulated in terms of norm residues.

A given rational number $a$ assigns to every prime spot $p$ a $p$-adic number $a_{p}=I_{p}(a)$. On which features of this assignment does one rely in forming the product (3)? The obvious answer is given by Chevalley's notion of idele: an idèle $\boldsymbol{a}$ is a function assigning to every prime spot $p$ a $p$-adic number $a_{p} \neq 0$ which is a $p$-adic unit for almost all prime spots $p$. The idèles form a multiplicative group $J_{k}$. By virtue of the assignment $a_{p}=I_{p}(a)$ every rational number $a \neq 0$ gives rise to an idèle, called the principal idele $a$. With the idèles $a$ at our disposal we might as well return to the notation $\left(\frac{a_{1} K}{p}\right)$ for $\left(a_{p}, K\right)$. The formula

$$
\phi_{K}(\mathbf{a})=(a, K)=\prod_{p}\left(a_{p}, K\right)=\prod_{p}\left(\frac{a, K}{p}\right)
$$

defines a character $\phi_{K}$, the norm character, in the group $J_{k}$ of all dèles. The reciprocity law in Hilbert's form (3) maintains that 


$$
(a, K)=1
$$

if $\boldsymbol{a}$ is principal. By the very definition of the norm symbol $\left(a_{p}, K\right)$ the same equation holds if $a$ is a norm in $K$, that is, if $a_{p}$ is a $p$-adic norm in $K$ for every prime spot $p$. Two idèles $a, a^{\prime}$ are said to be equivalent, $a \sim a^{\prime}$, if their quotient $\boldsymbol{a}^{\prime} \mathbf{a}^{-1}$ is principal. Let us denote by $\mathrm{Nm} J_{K}$ the group of all idèles which are equivalent to norms in $K$. Then (5) holds for all idèles a of $\mathrm{Nm} J_{K}$; it would be good to know that it holds for no other idèles, or, in other words, that $\mathrm{Nm} J_{K}$ is a subgroup of $J_{k}$ of index 2 .

The stage is now reached where the experiences gathered for a quadratic field $K$ over the rational ground field $k$ may be generalized to any relative Abelian field $K$ over a given algebraic number field $\kappa=k(\theta)$. First a word about the infinite prime spots of $\kappa$. The defining equation $f(\theta)=0$, an irreducible equation in $k$ of some degree $m$, has $m$ distinct roots $\theta^{\prime}, \theta^{\prime \prime}, \cdots, \theta^{(m)}$ in the continuum of complex numbers. Suppose that $r$ of them are real, say $\theta^{\prime}, \cdots, \theta^{(r)}$. Then each element $\alpha$ of $\kappa$ has its $r$ real conjugates $\alpha^{\prime}, \cdots, \alpha^{(r)}$, and $\alpha^{(t)}$ arises from $\alpha$ by a homomorphic projection $I^{(t)}$ of $\kappa$ into the field of all real numbers,

$$
\alpha \rightarrow \alpha^{(t)}=I^{(t)}(\alpha) \quad(t=1, \cdots, r) .
$$

We therefore speak of $r$ real infinite prime spots $\mathfrak{q}^{\prime}, \cdots, q^{(r)}$ with the corresponding projections $I^{\prime}=I_{q^{\prime}}, \cdots, I^{(r)}$; the fields $\kappa\left(\mathfrak{q}^{\prime}\right), \cdots$, $\kappa\left(q^{(r)}\right)$ are identical with the field of all real numbers. Thus $\alpha$ is an $n$th $q^{\prime}$-adic power if the equation $\alpha^{\prime}=\xi^{\prime n}$ has a real solution $\xi^{\prime}$. One sees that this imposes a condition only if $n$ is even, and then requires $\alpha^{\prime}$ to be positive. (In the complex domain the equation is always solvable whether $n$ be even or odd, and that is the reason why we ignore the complex infinite prime spots altogether.)

The finite prime spots are the prime ideals $\mathfrak{p}$ of $k$. In studying a Galois field $K / \kappa$ of relative degree $n$ we first exclude the ramified ideals $\mathfrak{p}$ which go into the relative discriminant of $K / \kappa$. An unramified ideal $\mathfrak{p}$ of $\kappa$ factors in $K$ into a number $g$ of distinct prime ideals $\mathfrak{B}_{1} \cdots \mathfrak{B}_{g}$ of relative degree $f, f g=n$. It is easily seen that a $p$-adic number $\alpha_{p} \neq 0$ is a $p$-adic norm in $K$ if and only if its order (at $\left.p\right)$ is a multiple of $f$. In particular, the $p$-adic units are norms. Thus we encounter a situation which is essentially simpler than the one taken care of by Gauss's quadratic residue symbol: the norm character of $\alpha_{p}$ depends only on the order $i$ at $p$ of $\alpha_{\mathfrak{p}}$. It is now clear how to proceed: we choose a primitive $f$ th root of unity $\zeta$ and define $\left(\alpha_{\mathfrak{p}}, K\right)=\zeta^{i}$ if $\alpha_{\mathfrak{p}}$ is of order $i$. This function of $\alpha_{p} \neq 0$ is a character which assumes the value 1 for the norms and the norms only. But here is the rub: there is no algebraic property distinguishing the several primitive $f$ th roots of unity 
from one another. Thus the choice of $\zeta$ among them remains arbitrary. One could put up with this if one dealt with one prime ideal only. But when one has to take all prime spots simultaneously into account, as is necessary in forming products of the type (4), then the arbitrariness involved in the choice of $\zeta$ for each $p$ will destroy all hope of obtaining a simple reciprocity law like (5). I shall forego describing the devices by which Eisenstein, Kummer, Hilbert, extricated themselves from this entanglement. By far the best solution was found by Artin: if $K / \kappa$ is Abelian, then the Frobenius substitution $\left(\frac{K}{p}\right)$ is uniquely determined by $K$ and $\mathfrak{p}$ and is an element of order $f$ of the Galois group $\Gamma$ of $K / \kappa$. Let this element of the Galois group replace $\zeta$ in our final definition of the $p$-adic norm symbol:

$$
\left(\alpha_{\mathfrak{p}}, K\right)=\left(\frac{\alpha, K}{\mathfrak{p}}\right)=\left(\frac{K}{\mathfrak{p}}\right)^{i} \text { if } \alpha_{\mathfrak{p}} \text { is of order } i \text { at } \mathfrak{p} .
$$

We could now form for any idèle $\alpha$ the product

$$
\prod_{\mathfrak{p}}\left(\alpha_{\mathfrak{p}}, K\right)=\prod_{\mathfrak{p}}\left(\frac{\boldsymbol{\alpha}, K}{\mathfrak{p}}\right)=(\boldsymbol{\alpha}, K)
$$

extending over all finite and (real) infinite prime spots $\mathfrak{p}$ and formulate the reciprocity law asserting that $(\boldsymbol{\alpha}, K)=1$ for any principal idèle $\boldsymbol{\alpha}$-had we not excluded certain exceptional prime spots in our definition of $\left(\alpha_{\mathfrak{p}}, K\right)$, namely the infinite prime spots and the ramified prime ideals. In the special case he investigated Kummer had succeeded in obtaining the correct value of $\left(\alpha_{\mathfrak{p}}, K\right)$ for the exceptional $\mathfrak{p}$ by extremely complicated calculations. Hilbert's fourth discovery is a simple and ingenious method of circumventing this formidable obstacle which blocked the road to further progress. Let us first restrict ourselves to idèles $\boldsymbol{\alpha}$ which are $n$th powers at our exceptional prime spots; in other words, we assume that the equation $\alpha_{\mathfrak{p}}=\xi_{\mathfrak{p}}^{n}$ is solvable for the $p$-adic values $\alpha_{\mathfrak{p}}$ of $\alpha$ at this finite number of prime spots. There is no difficulty in defining $(\boldsymbol{\alpha}, K)$ under this restriction:

$$
(\boldsymbol{\alpha}, K)=\prod_{\mathfrak{p}}^{\prime}\left(\alpha_{\mathfrak{p}}, K\right),
$$

the product extending, as indicated by the accent, over the non-exceptional prime spots only, for which we know what $\left(\alpha_{\mathfrak{p}}, K\right)$ means. Under the same restriction we prove (with Artin) the reciprocity law

$$
(\boldsymbol{\alpha}, K)=1 \text { if } \boldsymbol{\alpha} \text { is principal, }
$$

and observe that by its very definition $(\alpha, K)=1$ if $\alpha$ is norm. We 
now return to an arbitrary idèle $\alpha$. It is easily shown that there exists an equivalent idèle $\boldsymbol{\alpha}^{*} \sim \boldsymbol{\alpha}$ which is an $n$th power at all exceptional prime spots, but of course there will be plenty of them. However, the restricted law of reciprocity insures that

$$
\left(\alpha^{*}, K\right)=\prod_{\mathfrak{p}}^{\prime}\left(\alpha_{\mathfrak{p}}^{*}, K\right)
$$

has the same value for every one of the $\alpha^{* \prime} s$, and it is this value which we now denote by $(\alpha, K)$. This definition adopted, the reciprocity law (7) and the statement that $(\alpha, K)=1$ for every norm $\alpha$ follow at once without restriction. Thus the reciprocity law itself is made the tool for getting the exceptional prime spots under control!

Once $(\alpha, K)$ is known for every idèle $\alpha$ we can compute $\left(\alpha_{p}, K\right)$ for a given prime spot $\mathfrak{p}$ and a given $\mathfrak{p}$-adic number $\alpha_{\mathfrak{p}} \neq 0$ as the value of $(\alpha, K)$ for that "primary" idèle, also denoted by $\alpha_{\mathfrak{p}}$, which equals $\alpha_{\mathfrak{p}}$ at $\mathfrak{p}$ and 1 at any other prime spot. (The idèle $\alpha$ is the product of its primary components, $\boldsymbol{\alpha}=\prod_{p} \alpha_{\mathfrak{p}}$.) One expects the following two propositions to hold:

I. $\left(\alpha_{\mathfrak{p}}, K\right)=1$ if and only if $\alpha_{\mathfrak{p}}$ is a $\mathfrak{p}$-adic norm.

II. Given a prime ideal $\mathfrak{p},\left(\alpha_{\mathfrak{p}}, K\right)=1$ for every $\mathfrak{p}$-adic unit $\alpha_{\mathfrak{p}}$ if and only if $\mathfrak{p}$ is unramified.

The direct parts of I and II:

$\left(I_{0}\right) \alpha_{\mathfrak{p}}=$ norm implies $\left(\alpha_{\mathfrak{p}}, K\right)=1$;

$\left(I_{0}\right) \mathfrak{p}$ unramified implies $\left(\alpha_{\mathfrak{p}}, K\right)=1$ for every $\mathfrak{p}$-adic unit $\alpha_{\mathfrak{p}}$, were settled above. The converse statement of $I_{0}$ is trivial for the nonexceptional prime spots; but owing to the indirect definition of the norm symbol for the exceptional prime spots, the proofs of the converse of $\mathrm{I}_{0}$ for the exceptional spots and of the converse of $\mathrm{II}_{0}$ are rather intricate. From II we learn that for none of the ramified prime ideals $p$ does the norm character of $\alpha_{\mathfrak{p}}$ depend on the order of $\alpha_{\mathfrak{p}}$ only: this simple feature which makes the definition (6) possible is limited to non-ramified $\mathfrak{p}$. One would also expect:

III. If the principal idele $\alpha$ is an idele norm in $K$, then the number $\alpha$ is norm of a number in $K$.

This is true for cyclic fields $K / \kappa$, but in general not for Abelian fields.

Let us again denote by $\mathrm{Nm} J_{K}$ the subgroup in $J_{k}$ of the idèles which are equivalent to norms. Then the norm symbol $\phi_{K}(\alpha)=(\alpha, K)$ determines a homomorphic mapping of the factor group $J_{k} / \mathrm{Nm} J_{K}$ into the Galois group of $K / \kappa$. One would expect that this mapping is oneto-one: 
IV. By means of the norm symbol the factor group $J_{k} / \mathrm{Nm}_{K}$ is isomorphically mapped onto the Galois group of $K / \kappa$.

I, II, III ${ }_{c}$ (the subscript $c$ indicating restriction to cyclical fields) and IV are the main propositions of what one might call the norm theory of relative Abelian fields. They refer to a given field $K / \kappa$.

There is a second part of the theory, the class field theory proper, which is concerned with the manner in which all possible relative Abelian fields $K$ over $\kappa$ are reflected in the structure of the group $J_{*}$ of idèles in $\kappa$. Each such field $K$ determines, as we have seen, a subgroup $\mathrm{Nm} J_{K}$ of $J_{k}$ of finite index. The question arises which subgroups $J_{\kappa}^{*}$ of $J_{\kappa}$ are generated in this way by Abelian fields $K / \kappa$. Clearly the following conditions are necessary:

(1) Every principal idèle is in $J_{k}^{*}$.

(2) There is a natural number $n$ such that every $n$th power of an idèle is in $J_{k}^{*}$.

(3) There is a finite set $S$ of prime spots such that $\alpha$ is in $J_{k}^{*}$ provided $\alpha$ is a unit at every prime spot and equals 1 at the prime spots of $S$.

The main theorem concerning class fields states that these conditions are also sufficient.

V. Given a subgroup $J_{k}^{*}$ of $J_{k}$ fulfilling the above three conditions (and therefore, as one readily verifies, of finite index), there exists a uniquely determined Abelian field $K / \kappa$ such that $J_{k}^{*}=\mathrm{Nm} J_{K}$.

We divide the idèles of $\kappa$ into classes by throwing two ideles into the same class if their quotient is in $J_{k}^{*}$. Then $J_{k} / J_{k}^{*}$ is the class group and $K$ is called the corresponding class field. The most important example results if one lets $J_{k}^{*}$ consist of the unit ideles $\alpha$ whose values $\alpha_{p}$ are $p$-adic units at every prime spot $p .^{7}$ Then the classes may be described as the familiar classes of ideals: two ideals are put in the same class if their quotient is a principal ideal $(\alpha)$ springing from a number $\alpha$ positive at all real infinite prime spots. The corresponding class field $K$, the so-called absolute class field, is of relative discriminant 1 , and the largest unramified Abelian field over $\kappa$ (Theorem II). Its degree $n$ over $\kappa$ is the class number of ideals, its Galois group isomorphic to the class group of ideals in $\kappa$ (Theorem IV). $f$ being the least power of $\mathfrak{p}$ which lies in the principal class, $\mathfrak{p}$ decomposes into $n / f$ distinct prime ideals in $K$, each of relative degree $f$. This last statement does nothing but repeat the norm definition of the class field. Hence the way in which $\mathfrak{p}$ factors in $K$ depends only on the class to which $\mathfrak{p}$ belongs. The easiest way of extending the theory from the case with no ramified prime ideals, which was preponderant in Hilbert's thought,

\footnotetext{
${ }^{7}$ At the (real) infinite prime spots the positive numbers are considered the units.
} 
to Takagi's ramified case is by substituting idèles for ideals. Hilbert also stated that every ideal in $\kappa$ becomes a principal ideal in the absolute class field. It is today possible to show that this is so, by arguments, however, which are far from being fully understood, because this question transcends the domain of Abelian fields.

As was stated above, Hilbert did not prove these theorems in their full generality, but taking his departure from Gauss's theory of genera in quadratic fields and Kummer's investigations he worked his way gradually up from the simplest cases, developing as he went along the necessary machinery of new concepts and propositions about them until he could survey the whole landscape of class fields. We cannot attempt here to give an idea of the highly involved proofs. The completion of the work he left to his successors. The day is probably still far off when we shall have a theory of relative Galois number fields of comparable completeness.

Kronecker had shown, and Hilbert found a simpler proof for the fact, that Abelian fields over the rational ground field $k$ are necessarily subfields of the cyclotomic fields, and are thus obtained from the transcendental function $e^{2 \pi i x}$ by substituting rational values for the argument $x$. For Abelian fields over an imaginary quadratic field the so-called complex multiplication of the elliptic and modular functions plays a similar role ("Kronecker's Jugendtraum"). While Heinrich Weber following in Kronecker's footsteps, and R. Fuëter under Hilbert's guidance, made this dream come true, Hilbert himself began to play with modular functions of several variables which are defined by means of algebraic number fields, and to study their arithmetical implications. He never published these investigations, but $O$. Blumenthal, and later E. Hecke, used his notes and developed his ideas. The results are provocative, but still far from complete. It is indicative of the fertility of Hilbert's mind at this most productive period of his life that he handed over to his pupils a complex of problems of such fascination as that of the relation between number theory and modular functions. ${ }^{8}$

There remain to be mentioned a particularly simple proof of the transcendence of $e$ and $\pi$ with which Hilbert opened the series of his arithmetical papers, and the 1909 paper settling Waring's century-old conjecture. I should classify the latter paper among his most original ones, but we can forego considering it more closely because a decade later Hardy and Littlewood found a different approach which yields

${ }^{8}$ R. Fuëter, Singuläre Moduln und complexe Multiplication, 2 vols., Leipzig, 1924, 1927; cf. also H. Hasse, J. Reine Angew. Math. vol. 157 (1927) pp. 115-139. O. Blumenthal, Math. Ann. vol. 56 (1903) pp. 509-548, vol. 58 (1904) pp. 497-527. E. Hecke, Math. Ann. vol. 71 (1912) pp. 1-37, vol. 74 (1913) pp. 465-510. 
asymptotic formulas for the number of representations, and it is the Hardy-Littlewood "circle method" which has given rise in recent times to a considerable literature on this and related subjects. ${ }^{9}$

\section{Axiomatics}

There could not have been a more complete break than the one dividing Hilbert's last paper on the theory of number fields from his classical book, Grundlagen der Geometrie, published in 1899. Its only forerunner is a note of the year 1895 on the straight line as the shortest way. But O. Blumenthal records that as early as 1891 Hilbert, discussing a paper on the role of Desargues's and Pappus's theorems read by $\mathrm{H}$. Wiener at a mathematical meeting, made a remark which contains the axiomatic standpoint in a nutshell: "It must be possible to replace in all geometric statements the words point, line, plane, by table, chair, mug."

The Greeks had conceived of geometry as a deductive science which proceeds by purely logical processes once the few axioms have been established. Both Euclid and Hilbert carry out this program. However, Euclid's list of axioms was still far from being complete; Hilbert's list is complete and there are no gaps in the deductions. Euclid tried to give a descriptive definition of the basic spatial objects and relations with which the axioms deal; Hilbert abstains from such an attempt. All that we must know about those basic concepts is contained in the axioms. The axioms are, as it were, their implicit (necessarily incomplete) definitions. Euclid believed the axioms to be evident; his concern is the real space of the physical universe. But in the deductive system of geometry the evidence, even the truth of the axioms, is irrelevant; they figure rather as hypotheses of which one sets out to develop the logical .consequences. Indeed there are many different material interpretations of the basic concepts for which the axioms become true. For instance, the axioms of $n$-dimensional Euclidean vector geometry hold if a distribution of direct current in a given electric circuit, the $n$ branches of which connect in certain branching points, is called a vector, and Joule's heat produced per unit time by the current is considered the square of the vector's length. In building up geometry on the foundation of its axioms one will attempt to economize as much as possible and thus illuminate the role of the several groups of axioms. Arranged in their natural

- It must suffice here to quote the first paper in this line: G. H. Hardy and J. E. Littlewood, Quart. J. Math. vol. 48 (1919) pp. 272-293, and its latest successor which carries Waring's theorem over to arbitrary algebraic fields: C. L. Siegel, Amer. J. Math. vol. 66 (1944) pp. 122-136. 
hierarchy they are the axioms of incidence, order, congruence, parallelism, and continuity. For instance, if the theory of geometric proportions or of the areas of polygons can be established without resorting to the axioms of continuity, this ought to be done.

In all this, though the execution shows the hand of a master, Hilbert is not unique. An outstanding figure among his predecessors is M. Pasch, who had indeed traveled a long way from Euclid when he brought to light the hidden axioms of order and with methodical clarity carried out the deductive program for projective geometry (1882). Others in Germany (F. Schur) and a flourishing school of Italian geometers (Peano, Veronese) had taken up the discussion. With respect to the economy of concepts, Hilbert is more conservative than the Italians: quite deliberately he clings to the Euclidean tradition with its three classes of undefined elements, points, lines, planes, and its relations of incidence, order and congruence of segments and angles. This gives his book a peculiar charm, as if one looked into a face thoroughly familiar and yet sublimely transfigured.

It is one thing to build up geometry on sure foundations, another to inquire into the logical structure of the edifice thus erected. If I am not mistaken, Hilbert is the first who moves freely on this higher "metageometric" level: systematically he studies the mutual independence of his axioms and settles the question of independence from certain limited groups of axioms for some of the most fundamental geometric theorems. His method is the construction of models: the model is shown to disagree with one and to satisfy all other axioms; hence the one cannot be a consequence of the others. One outstanding example of this method had been known for a considerable time, the Cayley-Klein model of non-Euclidean geometry. For Veronese's nonArchimedean geometry Levi-Civita (shortly before Hilbert) had constructed a satisfactory arithmetical model. The question of consistency is closely related to that of independence. The general ideas appear to us almost banal today, so thoroughgoing has been their influence upon our mathematical thinking. Hilbert stated them in clear and unmistakable language, and embodied them in a work that is like a crystal: an unbreakable whole with many facets. Its artistic qualities have undoubtedly contributed to its success as a masterpiece of science.

In the construction of his models Hilbert displays an amazing wealth of invention. The most interesting examples seem to me the one by which he shows that Desargues's theorem does not follow from the plane incidence axioms, but that the plane incidence axioms combined with Desargues's theorem enable one to embed the plane in a higher dimensional space in which all incidence axioms hold; and then 
the other example by which he decides whether the Archimedean axiom of continuity is necessary to restore the full congruence axioms after having curtailed them by the exclusion of reflections.

What is the building material for the models? Klein's model of nonEuclidean geometry could be interpreted as showing that he who accepts Euclidean geometry with its points and lines, and so on, can by mere change of nomenclature also get the non-Euclidean geometry. Klein himself preferred another interpretation in terms of projective space. However, Descartes's analytic geometry had long provided a more general and satisfactory answer, of which Riemann, Klein and many others must have been aware: All that we need for our construction is the field of real numbers. Hence any contradiction in Euclidean geometry must show up as a contradiction in the arithmetical axioms on which our operations with real numbers are based. Nobody had said that quite clearly before Hilbert. He formulates a complete and simple set of axioms for real numbers. The system of arithmetical axioms will have its exchangeable parts just as the geometric system has. From a purely algebraic standpoint the most important axioms are those characterizing a (commutative or noncommutative) field. Any such abstract number field may serve as a basis for the construction of corresponding geometries. Vice versa, one may introduce numbers and their operations in terms of a space satisfying certain axioms; Hilbert's Desarguesian Streckenrechnung is a fine example. In general this reverse process is the more difficult one. The Chicago school under E. H. Moore took up Hilbert's investigations, and in particular $\mathrm{O}$. Veblen did much to reveal the perfect correspondence between the projective spaces obeying a set of simple incidence axioms (and no axioms of order), and the abstractly defined number fields. ${ }^{10}$

What the question of independence literally asks is to make sure that a certain proposition cannot be deduced from other propositions. It seems to require that we make the propositions, rather than the things of which they speak, the object of our investigation, and that as a preliminary we fully analyze the logical mechanism of deduction. The method of models is a wonderful trick to avoid that sort of logical investigations. It pays, however, a heavy price for thus shirking the fundamental issue: it merely reduces everything to the question of consistency for the arithmetical axioms, which is left unanswered. In

${ }^{10}$ Among later contributions to this question I mention W. Schwan, Streckenrechnung und Gruppentheorie, Math. Zeit. vol. 3 (1919) pp. 11-28. A complete bibliography of geometric axiomatics since Hilbert would probably cover many pages. I refrain from citing a list of names. 
the same manner completeness, which literally means that every general proposition about the objects with which the axioms deal can be decided by inference from the axioms, is replaced by categoricity (Veblen), which asserts that any imaginable model is isomorphic to the one model by which consistency is established. In this sense Hilbert proves that there is but "one," the Cartesian geometry, which fulfills all his axioms. Only in the case of G. Fano's and O. Veblen's finite projective spaces, for example, of the projective plane consisting of seven points, the model is a purely combinatorial scheme, and the questions of consistency, independence and completeness can be answered in the absolute sense. Hilbert never seems to have thought of illustrating his conception of the axiomatic method by purely combinatorial schemes, and yet they provide by far the simplest examples.

An approach to the foundations of geometry entirely different from the one followed in his book is pursued by Hilbert in a paper which is one of the earliest documents of set-theoretic topology. From the standpoint of mechanics the central task which geometry ought to perform is that of describing the mobility of a solid. This was the viewpoint of Helmholtz, who succeeded in characterizing the group of motions in Euclidean space by a few simple axioms. The question had been taken up by Sophus Lie in the light of his general theory of continuous groups. Lie's theory depends on certain assumptions of differentiability; to get rid of them is one of Hilbert's Paris Problems. In the paper just mentioned he does get rid of them as far as Helmholtz's problem in the plane is concerned. The proof is difficult and laborious; naturally continuity is now the foundation-and not the keystone of the building as it had been in his Grundlagen book. Other authors, R. L. Moore, N. J. Lennes, W. Süss, B. v. Kérékjarto, carried the problem further along these topological lines. A half-personal reminiscence may be of interest. Hilbert defines a two-dimensional manifold by means of neighborhoods, and requires that a class of "admissible" one-to-one mappings of a neighborhood upon Jordan domains in an $x, y$-plane be designated, any two of which are connected by continuous transformations. When I gave a course on Riemann surfaces at Göttingen in 1912, I consulted Hilbert's paper and noticed that the neighborhoods themselves could be used to characterize that class. The ensuing definition was given its final touch by F. Hausdorff; the Hausdorff axioms have become a byword in topology. ${ }^{11}$ (However, when it comes to explaining what a differenti-

11 A parallel development, with E. H. Moore as the chief prompter, must have taken place in this country. As I have to write from memory mainly, it is inevitable that my account should be colored by the local Göttingen tradition. 
able manifold is, we are to this day bound to Hilbert's roundabout way; cf. Veblen and Whitehead, The foundations of differential geometry, Cambridge, 1932.)

The fundamental issue of an absolute proof of consistency for the axioms which should include the whole of mathematical analysis, nay even Cantor's set theory in its wildest generality, remained in Hilbert's mind, as a paper read before the International Congress at Heidelberg in 1904 testifies. It shows him on the way, but still far from the goal. Then came the time when integral equations and later physics became his all-absorbing interest. One hears a loud rumbling of the old problem in his $Z$ ürich address, Axiomatisches Denken, of 1917. Meanwhile the difficulties concerning the foundations of mathematics had reached a critical stage, and the situation cried for repair. Under the impact of undeniable antinomies in set theory, Dedekind and Frege had revoked their own work on the nature of numbers and arithmetical propositions, Bertrand Russell had pointed out the hierarchy of types which, unless one decides to "reduce" them by sheer force, undermine the arithmetical theory of the continuum; and finally L. E. J. Brouwer by his intuitionism had opened our eyes and made us see how far generally accepted mathematics goes beyond such statements as can claim real meaning and truth founded on evidence. I regret that in his opposition to Brouwer, Hilbert never openly acknowledged the profound debt which he, as well as all other mathematicians, owe Brouwer for this revelation.

Hilbert was not willing to make the heavy sacrifices which Brouwer's standpoint demanded, and he saw, at least in outline, a way by which the cruel mutilation could be avoided. At the same time he was alarmed by signs of wavering loyalty within the ranks of mathematicians some of whom openly sided with Brouwer. My own article on the Grundlagenkrise in Math. Zeit. vol. 10 (1921), written in the excitement of the first postwar years in Europe, is indicative of the mood. Thus Hilbert returns to the problem of foundations in earnest. $\mathrm{He}$ is convinced that complete certainty can be restored without "committing treason to our science." There is anger and determination in his voice when he proposes "die Grundlagenfragen einf ürallemal aus der Welt zu schaffen." "Forbidding a mathematician to make use of the principle of excluded middle," says he, "is like forbidding an astronomer his telescope or a boxer the use of his fists."

Hilbert realized that the mathematical statements themselves could not be made the subject of a mathematical investigation whose aim is to answer the question of their consistency in its primitive 
sense, lest they be first reduced to mere formulas. Algebraic formulas like $a+b=b+a$ are the most familiar examples. The process of deduction by which formulas previously obtained give rise to new formulas must be described without reference to any meaning of the formulas. The deduction starts from certain initial formulas, the axioms, which must be written out explicitly. Whereas in his Grundlagen der Geometrie the meaning of the geometric terms had become irrelevant, but the meaning of logical terms, as "and," "not," "if then," had still to be understood, now every trace of meaning is obliterated. As a consequence, logical symbols like $\rightarrow$ in $a \rightarrow b$, read: $a$ implies $b$, enter into the formulas. Hilbert fully agrees with Brouwer in that the great majority of mathematical propositions are not "real" ones conveying a definite meaning verifiable in the light of evidence. But he insists that the non-real, the "ideal propositions" are indispensable in order to give our mathematical system "completeness." Thus he parries Brouwer, who had asked us to give up what is meaningless, by relinquishing the pretension of meaning altogether, and what he tries to establish is not truth of the individual mathematical proposition, but consistency of the system. The game of deduction when played according to rules, he maintains, will never lead to the formula $0 \neq 0$. In this sense, and in this sense only, he promises to salvage our cherished classical mathematics in its entirety.

For those who accuse him of degrading mathematics to a mere game he points first to the introduction of ideal elements for the sake of completeness as a common method in all mathematics-for example, of the ideal points outside an accessible portion of space, without which space would be incomplete-; secondly, to the neighboring science of physics where likewise not the individual statement is verifiable by experiment, but in principle only the system as a whole can be confronted with experience.

But how to make sure that the "game of deduction" never leads to a contradiction? Shall we prove this by the same mathematical method the validity of which stands in question, namely by deduction from axioms? This would clearly involve a regress ad infinitum. It must have been hard on Hilbert, the axiomatist, to acknowledge that the insight of consistency is rather to be attained by intuitive reasoning which is based on evidence and not on axioms. But after all, it is not surprising that ultimately the mind's seeing eye must come in. Already in communicating the rules of the game we must count on understanding. The game is played in silence, but the rules must be told and any reasoning about it, in particular about its consistency, communicated by words. Incidentally, in describing the indispensable 
intuitive basis for his Beweistheorie Hilbert shows himself an accomplished master of that, alas, so ambiguous medium of communication, language. With regard to what he accepts as evident in this "metamathematicial" reasoning, Hilbert is more papal than the pope, more exacting than either Kronecker or Brouwer. But it cannot be helped that our reasoning in following a hypothetic sequence of formulas leading up to the formula $0 \neq 0$ is carried on in hypothetic generality and uses that type of evidence which a formalist would be tempted to brand as application of the principle of complete induction. Elementary arithmetics can be founded on such intuitive reasoning as Hilbert himself describes, but we need the formal apparatus of variables and "quantifiers" to invest the infinite with the all important part that it plays in higher mathematics. Hence Hilbert prefers to make a clear cut: he becomes strict formalist in mathematics, strict intuitionist in metamathematics.

It is perhaps possible to indicate briefly how Hilbert's formalism restores the principle of the excluded middle which was the main target of Brouwer's criticism. Consider the infinite sequence of numbers $0,1,2, \cdots$. Any property $A$ of numbers (for example, "being prime") may be represented by a propositional function $A(x)$ (" $x$ is prime"), from which a definite proposition $A(b)$ arises by substituting a concrete number $b$ for the variable $x$ (" 6 is prime"). Accepting the principle which Brouwer denies and to which Hilbert wishes to hold on, that (i) either there exists a number $x$ for which $A(x)$ holds, or (ii) $A(x)$ holds for no $x$, we can find a "representative" $r$ for the property $A$, a number such that $A(b)$ implies $A(r)$ whatever the number $b$, $A(b) \rightarrow A(r)$. Indeed, in the alternative (i) we choose $r$ as one of the numbers $x$ for which $A(x)$ holds, in the alternative (ii) at random. Thus Aristides is the representative of honesty; for, as the Athenians said, if there is any honest man it is Aristides. Assuming that we know the representative we can decide the question whether there is an honest man or whether all are dishonest by merely looking at him: if he is dishonest everybody is. In the realm of numbers we may even make the choice of the representative unique, in case (i) choosing $x=r$ as the least number for which $A(x)$ holds, and $r=0$ in the opposite case (ii). Then $r$ arises from $A$ by a certain operator $\rho_{x}$, $r=\rho_{x} A(x)$, applicable to every imaginable property $A$. A propositional function may contain other variables $y, z, \cdots$ besides $x$. Therefore it is necessary to attach an index $x$ to $\rho$, just as in integrating one must indicate with respect to which variable one integrates. $\rho_{x}$ eliminates the variable $x$; for instance $\rho_{x} A(x, y)$ is a function of $y$ alone. The word quantifier is in use for this sort of operator. Hence 
we write our axiom as follows:

$$
A(b) \rightarrow A\left(\rho_{x} A(x)\right) .
$$

It is immaterial whether we fix the representative in the unique manner described above; our specific rule would not fit anyhow unless $x$ ranges over the numbers $0,1,2, \cdots$. Instead we imagine a quantifier $\rho_{x}$ of universal applicability which, as it were, selects the representative for us. Zermelo's axiom of choice is thus woven into the principle of the excluded middle. It is a bold step; but the bolder the better, as long as it can be shown that we keep within the bounds of consistency!

In the formalism, propositional functions are replaced by formulas the handling of which must be described without reference to their meaning. In general, variables $x, y, \cdots$ will occur among the symbols of a formula $\mathfrak{A}$. We say that the symbol $\rho_{x}$ binds the variable $x$ in the formula $\mathfrak{A}$ which follows the symbol ${ }^{12}$ and that $x$ occurs free in a formula wherever it is not bound by a quantifier with index $x$. $x, y, \rightarrow, \rho_{x}$ are symbols entering into the formulas; the German letters are no such symbols, but are used for communication. It is more natural to describe our critical axiom (8) as a rule for the formation of axioms. It says: take any formula $\mathfrak{A}$ in which only the variable $x$ occurs free, and any formula $\mathfrak{b}$ without free variables, and by means of them build the formula

$$
\mathfrak{A}(\mathfrak{b}) \rightarrow \mathfrak{A}\left(\rho_{x} \mathfrak{Z}\right) .
$$

Here $\mathfrak{A}(\mathfrak{b})$ stands for the formula derived from $\mathfrak{A}$ by putting in the entire formula $\mathfrak{b}$ for the variable $x$ wherever $x$ occurs free.

In this way formulas may be obtained as axioms according to certain rules. Deduction proceeds by the rule of syllogism: From two formulas $\mathfrak{a}$ and $\mathfrak{a} \rightarrow \mathfrak{b}$ previously obtained, in the second of which the first formula reappears at the left of the symbol $\rightarrow$, one obtains the formula $\mathfrak{b}$.

How does Hilbert propose to show that the game of deduction will never lead to the formula $0 \neq 0$ ? Here is the basic idea of his procedure. As long as one deals with "finite" formulas only, formulas from which the quantifiers $\rho_{x}, \rho_{y}, \cdots$ are absent, one can decide whether they are true or false by merely looking at them. With the entrance of $\rho$ such a descriptive valuation of formulas becomes impossible: evidence ceases to work. But a concretely given deduction is a sequence of

12 If we wish the rule that $\rho_{x}$ binds $x$ in all that comes after to be taken literally, we must write $\mathfrak{a} \rightarrow \mathfrak{b}$ in the form $\rightarrow\{\mathfrak{a} \mathfrak{b}$. The formulas will then look like genealogical trees. 
formulas in which only a limited number of instances of the axiomatic rule (9) will turn up. Let us assume that the only quantifier which occurs is $\rho_{x}$ and wherever it occurs it is followed by the same finite formula $\mathfrak{A}$, so that the instances of (9) are of the form

$$
\mathfrak{A}\left(\mathfrak{b}_{1}\right) \rightarrow \mathfrak{A}\left(\rho_{x} \mathfrak{A}\right), \cdots, \mathfrak{A}\left(\mathfrak{b}_{h}\right) \rightarrow \mathfrak{A}\left(\rho_{x} \mathfrak{V}\right) .
$$

Assume, moreover, $\mathfrak{b}_{1}, \cdots, \mathfrak{b}_{h}$ to be finite. We then carry out a reduction, replacing $\rho_{x} \mathfrak{I}$ by a certain finite $\mathfrak{r}$ wherever it occurs as part of a formula in our sequence. In particular, the formulas (10) will change into

$$
\mathfrak{A}\left(\mathfrak{b}_{1}\right) \rightarrow \mathfrak{A}(\mathfrak{r}), \cdots, \mathfrak{A}\left(\mathfrak{b}_{h}\right) \rightarrow \mathfrak{A}(\mathfrak{r}) .
$$

We now see how to choose $\mathfrak{r}$ : if by examining the finite formulas $\mathfrak{A}\left(\mathfrak{b}_{1}\right), \cdots, \mathfrak{A}\left(\mathfrak{b}_{k}\right)$ one after the other, we find one that is true, say $\mathfrak{U}\left(\mathfrak{b}_{3}\right)$, then we take $\mathfrak{b}_{3}$ for $\mathfrak{r}$. If every one of them turns out to be false, we choose $\mathfrak{r}$ at random. Then the $h$ reduced formulas (11) are "true" and our hypothesis that the deduction leads to the false formula $0 \neq 0$ is carried ad absurdum. The salient point is that a concretely given deduction makes use of a limited number of explicitly exhibited individuals $\mathfrak{b}_{1}, \cdots, \mathfrak{b}_{h}$ only. If we make a wrong choice, for example, by choosing Alcibiades rather than Aristides as the representative of incorruptibility, our mistake will do no harm as long as the few people (out of the infinite Athenian crowd) with whom we actually deal are all corruptible.

A slightly more complicated case arises when we permit the $\mathfrak{b}_{1}, \cdots, \mathfrak{b}_{h}$ to contain $\rho_{x}$, but always followed by the same $\mathfrak{A}$. Then we first make a tentative reduction replacing $\rho_{x} \mathfrak{A}$ by the number 0 , say. The formulas $\mathfrak{b}_{1}, \cdots, \mathfrak{b}_{h}$ are thus changed into reduced finite formulas $\mathfrak{b}_{1}^{0}, \cdots, \mathfrak{b}_{h}^{0}$ and (10) into

$$
\mathfrak{A}\left(\mathfrak{b}_{1}^{0}\right) \rightarrow \mathfrak{A}(0), \cdots, \mathfrak{A}\left(\mathfrak{b}_{h}^{0}\right) \rightarrow \mathfrak{A}(0) .
$$

This reduction will do unless $\mathscr{A}(0)$ is false and at the same time one of the $\mathfrak{A}\left(\mathfrak{b}_{1}^{0}\right), \cdots, \mathfrak{A}\left(\mathfrak{b}_{\mathfrak{h}}^{0}\right)$, say $\mathfrak{A}\left(\mathfrak{b}_{3}^{0}\right)$, is true. But then we have in $\mathfrak{b}_{3}^{0}$ a perfectly legitimate representative of $\mathfrak{A}$, and a second reduction which replaces $\rho_{x} \mathfrak{A}$ by $\mathfrak{b}_{3}^{0}$ will work out all right.

However, this is only a modest beginning of the complications awaiting us. Quantifiers $\rho_{x}, \rho_{y}, \ldots$ with different variables and applied to different formulas will be piled one upon the other. We make a tentative reduction; it will go wrong in certain places and from that failure we learn how to correct it. But the corrected reduction will probably go wrong at other places. We seem to be driven around in a vicious circle, and the problem is to direct our consecutive corrections 
in such a manner as to obtain assurance that finally a reduction will result that makes good at all places in our given sequence of formulas. Nothing has contributed more to revealing the circle-like character of the usual transfinite arguments of mathematics than these attempts to make sure of consistency in spite of all circles.

The symbolism for the formalization of mathematics as well as the general layout and first steps of the proof of consistency are due to Hilbert himself. The program was further advanced by younger collaborators, P. Bernays, W. Ackermann, J. von Neumann. The last two proved the consistency of "arithmetics," of that part in which the dangerous axiom about the conversion of predicates into sets is not yet admitted. A gap remained which seemed harmless at the time, but already detailed plans were drawn up for the invasion of analysis. Then came a catastrophe: assuming that consistency is established, K. Gödel showed how to construct arithmetical propositions which are evidently true and yet not deducible within the formalism. His method applies to Hilbert's as well as any other not too limited formalism. Of the two fields, the field of formulas obtainable in Hilbert's formalism and the field of real propositions that are evidently true, neither contains the other (provided consistency of the formalism can be made evident). Obviously completeness of a formalism in the absolute sense in which Hilbert had envisaged it was now out of the question. When G. Gentzen later closed the gap in the consistency proof for arithmetics, which Göbel's discovery had revealed to be serious indeed, he succeeded in doing so only by substantially lowering Hilbert's standard of evidence. ${ }^{13}$ The boundary line of what is intuitively trustworthy once more became vague. As all hands were needed to defend the homeland of arithmetics, the invasion of analysis never came off, to say nothing of general set theory.

This is where the problem now stands; no final solution is in sight. But whatever the future may bring, there is no doubt that Brouwer and Hilbert raised the problem of the foundations of mathematics to a new level. A return to the standpoint of Russell-Whitehead's Principia Mathematica is unthinkable.

Hilbert is the champion of axiomatics. The axiomatic attitude seemed to him one of universal significance, not only for mathematics, but for all sciences. His investigations in the field of physics are conceived in the axiomatic spirit. In his lectures he liked to illustrate the method by examples taken from biology, economics, and so on. The modern epistemological interpretation of science has been profoundly influenced by him. Sometimes when he praised the axiomatic method

${ }^{18}$ G. Gentzen, Math. Ann. vol. 112 (1936) pp. 493-565. 
he seemed to imply that it was destined to obliterate completely the constructive or genetic method. I am certain that, at least in later life, this was not his true opinion. For whereas he deals with the primary mathematical objects by means of the axioms of his symbolic system, the formulas are constructed in the most explicit and finite manner. In recent times the axiomatic method has spread from the roots to all branches of the mathematical tree. Algebra, for one, is permeated from top to bottom by the axiomatic spirit. One may describe the role of axioms here as the subservient one of fixing the range of variables entering into the explicit constructions. But it would not be too difficult to retouch the picture so as to make the axioms appear as the masters. An impartial attitude will do justice to both sides; not a little of the attractiveness of modern mathematical research is due to a happy blending of axiomatic and genetic procedures.

\section{INTEGRAL EQUATIONS}

Between the two periods during which Hilbert's efforts were concentrated on the foundations, first of geometry, then of mathematics in general, there lie twenty long years devoted to analysis and physics.

In the winter of 1900-1901 the Swedish mathematician E. Holmgren reported in Hilbert's seminar on Fredholm's first publications on integral equations, and it seems that Hilbert caught fire at once. The subject has a long and tortuous history, beginning with Daniel Bernoulli. The mathematicians' efforts to solve the (mechanical, acoustical, optical, electromagnetical) problem of the oscillations of a continuum and the related boundary value problems of potential theory span a period of two centuries. Fourier's Theorie analytique de la chaleur (1822) is a landmark. H. A. Schwarz proved for the first time (1885) the existence of a proper oscillation in two and more dimensions by constructing the fundamental frequency of a membrane. The last decade of the nineteenth century saw Poincare on his way to the development of powerful function-theoretic methods; C. Neumann and he came to grips with the harmonic boundary problem; Volterra studied that type of integral equations which now bears his name, and for linear equations with infinitely many unknowns Helge von Koch developed the infinite determinants. Most scientific discoveries are made when "their time is fulfilled"; sometimes, but seldom, a genius lifts the veil decades earlier than could have been expected. Fredholm's discovery has always seemed to me one that was long overdue when it came. What could be more natural than the idea that a set of linear equations connected with a discrete set of mass points gives way to an integral equation when one passes to 
the limit of a continuum? But the fact that in the simpler cases a differential rather than an integral equation results in the limit riveted the mathematicians' attention for two hundred years on differential equations!

It must be said, however, that the simplicity of Fredholm's results is due to the particular form of his equation, on which it was hard to hit without the guidance of the problems of mathematical physics to which he applied it:

$$
x(s)-\int_{0}^{1} K(s, t) x(t) d t=f(s) \quad(0 \leqq s \leqq 1) .
$$

Indeed the linear operator which in the left member operates on the unknown function $x$ producing a given $f,(E-K) x=f$, consists of two parts, the identity $E$ and the integral operator $K$, which in a certain sense is weak compared to $E$. Fredholm proved that for this type of integral equation the two main facts about $n$ linear equations with the same number $n$ of unknowns hold: (1) The homogeneous equation $[f(s)=0]$ has a finite number of linearly independent solutions $x(s)=\phi_{1}(s), \cdots, \phi_{h}(s)$, and the homogeneous equation with the transposed kernel $K^{\prime}(s, t)=K(t, s)$ has the same number of solutions, $\psi_{1}(s), \cdots, \psi_{h}(s)$. (2) The nonhomogeneous equation is solvable if and only if the given $f$ satisfies the $h$ linear conditions

$$
\int_{0}^{1} f(s) \psi_{i}(s) d s=0 \quad(i=1, \cdots, h) .
$$

Following an artifice used by Poincaré, Fredholm introduces a parameter $\lambda$ replacing $K$ by $\lambda K$ and obtains a solution in the form familiar from finite linear equations, namely as a quotient of two determinants of H. v. Koch's type, either of which is an entire function of the parameter $\lambda$.

Hilbert saw two things: (1) after having constructed Green's function $K$ for a given region $G$ and for the potential equation $\Delta u=0$ by means of a Fredholm equation on the boundary, the differential equation of the oscillating membrane $\Delta \phi+\lambda \phi=0$ changes into a homogeneous integral equation

$$
\phi(s)-\lambda \int K(s, t) \phi(t) d t=0
$$

with the symmetric kernel $K, K(t, s)=K(s, t)$ (in which the parameter $\lambda$ is no longer artificial but of the very essence of the problem); (2) the problem of ascertaining the "eigen values" $\lambda$ and "eigen func- 
tions" $\phi(s)$ of this integral equation is the analogue for integrals of the transformation of a quadratic form of $n$ variables onto principal axes. Hence the corresponding theorem for the quadratic integral form

$$
\int_{0}^{1} \int_{0}^{1} K(s, t) x(s) x(t) d s d t
$$

with an arbitrary symmetric kernel $K$ must provide the general foundation for the theory of oscillations of a continuous medium. If others saw the same, Hilbert saw it at least that much more clearly that he bent all his energy on proving that proposition, and he succeeded by the same direct method which about 1730 Bernoulli had applied to the oscillations of a string: passage to the limit from the algebraic problem. In carrying out the limiting process he had to make use of the Koch-Fredholm determinant. He finds that there is a sequence of eigen values $\lambda_{1}, \lambda_{2}, \cdots$ tending to infinity, $\lambda_{n} \rightarrow \infty$ for $n \rightarrow \infty$, and an orthonormal set of corresponding eigen functions $\phi_{n}(s)$,

$$
\begin{gathered}
\phi_{n}(s)-\lambda_{n} \int_{0}^{1} K(s, t) \phi_{n}(t) d t=0, \\
\int_{0}^{1} \phi_{m}(s) \phi_{n}(s) d s=\delta_{m n},
\end{gathered}
$$

such that

$$
\int_{0}^{1} \int_{0}^{1} K(s, t) x(s) x(t) d s d t=\sum \xi_{n}^{2} / \lambda_{n}
$$

$\xi_{n}$ being the Fourier coefficient $\int_{0}^{1} x(s) \phi_{n}(s) d s$. The theory implies that every function of the form

$$
y(s)=\int_{0}^{1} K(s, t) x(t) d t
$$

may be expanded into a uniformly convergent Fourier series in terms of the eigen functions $\phi_{n}$,

$$
y(s)=\sum \eta_{n} \phi_{n}(s), \quad \eta_{n}=\int_{0}^{1} y(s) \phi_{n}(s) d s .
$$

Hilbert's passage to the limit is laborious. Soon afterwards E. Schmidt in a Göttingen thesis found a simpler and more constructive proof for these results by adapting H. A. Schwarz's method invented twenty years before to the needs of integral equations.

From finite forms the road leads either to integrals or to infinite 
series. Therefore Hilbert considered the same problem of orthogonal transformation of a given quadratic form

$$
\sum K_{m n} x_{m} x_{n}
$$

into a form of the special type

$$
\kappa_{1} \xi_{1}^{2}+\kappa_{2} \xi_{2}^{2}+\cdots \quad\left(\kappa_{n}=1 / \lambda_{n} \rightarrow 0\right)
$$

also for infinitely many (real) variables $\left(x_{1}, x_{2}, \cdots\right)$ or vectors $x$ in a space of a denumerable infinity of dimensions. Only such vectors are admitted as have a finite length $|x|$,

$$
|x|^{2}=x_{1}^{2}+x_{2}^{2}+\cdots ;
$$

they constitute what we now call the Hilbert space. The advantage of Hilbert space over the "space" of all continuous functions $x(s)$ lies in a certain property of completeness, and due to this property one can establish "complete continuity" as the necessary and sufficient condition for the transformability of a given quadratic form $K$, (13), into (14), by following an argument well known in the algebraic case: one determines $\kappa_{1}, \kappa_{2}, \cdots$ as the consecutive maxima of $K$ on the "sphere" $|x|^{2}=1$.

As suggested by the theorem concerning a quadratic integral form, the link between the space of functions $x(s)$ and the Hilbert space of vectors $\left(x_{1}, x_{2}, \cdots\right)$ is provided by an arbitrary complete orthonormal system $u_{1}(s), u_{2}(s), \cdots$ and expressed by the equations

$$
x_{n}=\int_{0}^{1} x(s) u_{n}(s) d s .
$$

Bessel's inequality states that the square sum of the Fourier coeffcients $x_{n}$ is less than or equal to the square integral of $x(s)$. The relation of completeness, first introduced by A. Hurwitz and studied in detail by W. Stekloff, requires that in this inequality the equality sign prevail. Thus the theorem on quadratic forms of infinitely many variables at once gives the corresponding results about the eigen values and eigen functions of symmetric kernels $K(s, t)$-or would do so if one could count on the uniform convergence of $\sum x_{n} u_{n}(s)$ for any given vector $\left(x_{1}, x_{2}, \cdots\right)$ in Hilbert space. In the special case of an eigen vector of that quadratic form (13) which corresponds to the integral form (12),

$$
x_{n}=\lambda \sum_{m} K_{n m} x_{m}
$$


Hilbert settles this point by forming the uniformly convergent series

$$
\lambda \sum_{m} x_{m} \int_{0}^{1} K(s, t) u_{m}(t) d t
$$

which indeed yields a continuous function $\phi(s)$ with the $n$th Fourier coefficient

$$
\lambda \sum K_{n m} x_{m}=x_{n},
$$

and thus obtains the eigen function of $K(s, t)$ for the eigen value $\lambda$. Soon afterwards, under the stimulus of Hilbert's investigations. E. Fischer and F. Riesz proved their well known theorem that the space of all functions $x(s)$ the square of which has a finite Lebesgue integral enjoys the same property of completeness as Hilbert space, and hence one is mapped isomorphically upon the other in a one-toone fashion by means of a complete orthonormal system $u_{n}(s)$. I mention these details because the historic order of events may have fallen into oblivion with many of our younger mathematicians, for whom Hilbert space has assumed that abstract connotation which no longer distinguishes between the two realizations, the square integrable functions $x(s)$ and the square summable sequences $\left(x_{1}, x_{2}, \cdots\right)$. I think Hilbert was wise to keep within the bounds of continuous functions when there was no actual need for introducing Lebesgue's general concepts.

Perhaps Hilbert's greatest accomplishment in the field of integral equations is his extension of the theory of spectral decomposition from the completely continuous to the so-called bounded quadratic forms. $\mathrm{He}$ finds that then the point spectrum will in general have condensation points and a continuous spectrum will appear beside the point spectrum. Again he proceeds by directly carrying out the transition to the limit, letting the number of variables $x_{1}, x_{2}, \ldots$ increase $a d$ infinitum. Again, not long afterwards, simpler proofs for his results were found.

While thus advancing the boundaries of the general theory, he did not lose sight of the ordinary and partial differential equations from which it had sprung. Simultaneously with the young Italian mathematician Eugenio Elia Levi he developed the parametrix method as a bridge between differential and integral equations. For a given elliptic differential operator $\Delta^{*}$ of the second order, the parametrix $K(s, t)$ is a sort of qualitative approximation of Green's function, depending like the latter on an argument point $s$ and a parameter point $t$. It is supposed to possess the right kind of singularity for $s=t$ so that the nonhomogeneous equation $\Delta^{*} u=f$ for 


$$
u=K \rho, \quad u(s)=\int K(s, t) \rho(t) d t
$$

gives rise to the integral equation $\rho+L \rho=f$ for the density $\rho$, with a kernel $L(s, t)=\Delta_{s}^{*} K(s, t)$ regular enough at $s=t$ for Fredholm's theory to be applicable. It is important to give up the assumption that $K$ satisfies the equation $\Delta^{*} K=0$, because in general a fundamental solution will not be known for the given differential operator $\Delta^{*}$. In order not to be bothered by boundary conditions, Hilbert assumes the domain of integration to be a compact manifold, like the surface of a sphere, and finds that the method works if the parametrix, besides having the right kind of singularity, is symmetric with respect to argument and parameter.

What has been said should be enough to make clear that in the terrain of analysis a rich vein of gold had been struck, comparatively easy to exploit and not soon to be exhausted. The linear equations of infinitely many unknowns had to be investigated further (E. Schmidt, F. Riesz, O. Toeplitz, E. Hellinger, and others); the continuous spectrum and its appearance in integral equations with "singular" kernels awaited closer analysis (E. Hellinger, T. Carleman); ordinary differential equations, with regular or singular boundaries, of second or of higher order, received their due share of attention (A. Kneser, E. Hilb, G. D. Birkhoff, M. Bôcher, J. D. Tamarkin, and many others). ${ }^{14}$ It became possible to develop such asymptotic laws for the distribution of eigen values as were required by the thermodynamics of radiation (H. Weyl, R. Courant). Expansions in terms of orthogonal functions were studied independently of their origin in differential or integral equations. New light fell upon Stieltjes's continued fractions and the problem of momentum. The most ambitious began to attack nonlinear integral equations. A large international school of young mathematicians gathered around Hilbert and integral equations became the fashion of the day, not only in Germany, but also in France where great masters like E. Picard and Goursat paid their tributes, in Italy and on this side of the Atlantic. Many good papers were written, and many mediocre ones. But the total effect was an appreciable change in the aspect of analysis.

Remarkable are the applications of integral equations outside the field for which they were invented. Among them I mention the following three: (1) Riemann's problem of determining $n$ analytic func-

14 For later literature and systems of differential equations see Axel Schur, Math. Ann. vol. 82 (1921) pp. 213-239; G. A. Bliss, Trans. Amer. Math. Soc. vol. 28 (1926) pp. 561-584; W. T. Reid, ibid. vol. 44 (1938) pp. 508-521. 
tions $f_{1}(z), \cdots, f_{n}(z)$, regular except at a finite number of points, which by analytic continuation around these points suffer preassigned linear transformations. The problem was solved by Hilbert himself, and subsequently in a simpler and more complete form by J. Plemelj. (A very special case of it is the existence of algebraic functions on a Riemann surface if that surface is given as a covering surface of the complex z-plane.) Investigations by G. D. Birkhoff on matrices of analytic functions lie in the same line. (2) Proof for the completeness of the irreducible representations of a compact continuous group. This is an indispensable tool for the approach to the general theory of invariants by means of Adolf Hurwitz's integration method, and with its refinements and generalizations plays an important role in modern group-theoretic research, including $\mathrm{H}$. Bohr's theory of almost periodic functions. ${ }^{15}$ Contact is thus made with Hilbert's old friend, the theory of invariants. (3) Quite recently Hilbert's parametrix method has served to establish the central existence theorem in W. V. D. Hodge's theory of harmonic integrals in compact Riemannian spaces. ${ }^{16}$

The story would be dramatic enough had it ended here. But then a sort of miracle happened: the spectrum theory in Hilbert space was discovered to be the adequate mathematical instrument of the new quantum physics inaugurated by Heisenberg and Schrödinger in 1923. This latter impulse led to a reexamination of the entire complex of problems with refined means (J. von Neumann, A. Wintner, M. H. Stone, K. Friedrichs). As J. von Neumann was Hilbert's collaborator toward the close of that epoch when his interest was divided between quantum physics and foundations, the historic continuity with Hilbert's own scientific activities is unbroken, even for this later phase. What has become of the theory of abstract spaces and their linear operators in our times lies beyond the bounds of this report.

A picture of Hilbert's "analytic" period would be incomplete without mentioning a second motif, calculus of variations, which crossed the dominating one of integral equations. The "theorem of independence" with which he concludes his Paris survey of mathematical problems (1900) is an important contribution to the formal apparatus of that calculus. But of much greater consequence was his audacious direct assault on the functional maxima and minima problems. The whole finely wrought machinery of the calculus of variations is here

${ }^{16} \mathrm{H}$. Weyl and F. Peter, Math. Ann. vol. 97 (1927) pp. 737-755. A. Haar, Ann. of Math. vol. 34 (1933) pp. 147-169. J.von Neumann, Trans. Amer. Math. Soc. vol. 36 (1934) pp. 445-492. Cf. also L. Pontrjagin, Topological groups, Princeton, 1939.

${ }^{16} \mathrm{~W}$. V. D. Hodge, The theory and applications of harmonic integrals, Cambridge, 1941. H. Weyl, Ann. of Math. vol. 44 (1943) pp. 1-6. 
consciously set aside. He proposes instead to construct the minimizing function as the limit of a sequence of functions for which the value of the integral under investigation tends to its minimum value. The classical example is Dirichlet's integral in a two-dimensional region $G$,

$$
D[u]=\iint_{G}\left\{\left(\frac{\partial u}{\partial x}\right)^{2}+\left(\frac{\partial u}{\partial y}\right)^{2}\right\} d x d y .
$$

Admitted are all functions $u$ with continuous derivatives which have given boundary values. $d$ being the lower limit of $D[u]$ for admissible $u$, one can ascertain a sequence of admissible functions $u_{n}$ such that $D\left[u_{n}\right] \rightarrow d$ with $n \rightarrow \infty$. One cannot expect the $u_{n}$ themselves to converge; rather they have to be prepared for convergence by the smoothing process of integration. As the limit function will be harmonic and the value of the harmonic function at any point $P$ equals its mean value over any circle $K$ around $P$, it seems best to replace $u_{n}(P)$ by its mean value in $K$, with the expectation that this mean value will converge toward a number $u(P)$ which is independent of the circle and in its dependence on $P$ solves the minimum problem. Besides integration Hilbert uses the process of sifting a suitable subsequence from the $u_{n}$ before passing to the limit. Owing to the simple inequality

$$
\left\{D\left[u_{m}-u_{n}\right]\right\}^{1 / 2} \leqq\left\{D\left[u_{m}\right]-d\right\}^{1 / 2}+\left\{D\left[u_{m}\right]-d\right\}^{1 / 2}
$$

discovered by S. Zaremba this second step is unnecessary.

Hilbert's method is even better suited for problems in which the boundary does not figure so prominently as in the boundary value problems. By a slight modification one is able to include point singularities, and Hilbert thus solved the fundamental problem for flows on Riemann surfaces, providing thereby the necessary foundation for Riemann's own approach to the theory of Abelian integrals, and he further showed that Poincaré's and Koebe's fundamental theorems on uniformization could be established in the same way. We should be much better off in number theory if methods were known which are as powerful for the construction of relative Abelian and Galois fields over given algebraic number fields as the Riemann-Hilbert transcendental method proves to be for the analogous problems in the fields of algebraic functions! Its wide application in the theory of conformal mapping and of minimal surfaces is revealed by the work of the man who was Hilbert's closest collaborator in the direction of mathematical affairs at Göttingen for many years, Richard Courant. ${ }^{17}$

${ }^{17} \mathrm{~A}$ book by Courant on the Dirichlet principle is in preparation. 
Of a more indirect character, but of considerable vigor, is the influence of Hilbert's ideas upon the whole trend of the modern development of the calculus of variations; in Europe Carathéodory, Lebesgue, Tonelli could be mentioned among others, in this country the chain reaches from $\mathrm{O}$. Bolza's early to $\mathrm{M}$. Morse's most recent work.

\section{Physics}

Already before Minkowski's death in 1909, Hilbert had begun a systematic study of theoretical physics, in close collaboration with his friend who had always kept in touch with the neighboring science. Minkowski's work on relativity theory was the first fruit of these joint studies. Hilbert continued them through the years, and between 1910 and 1930 often lectured and conducted seminars on topics of physics. He greatly enjoyed this widening of his horizon and his contact with physicists, whom he could meet on their own ground. The harvest however can hardly be compared with his achievements in pure mathematics. The maze of experimental facts which the physicist has to take into account is too manifold, their expansion too fast, and their aspect and relative weight too changeable for the axiomatic method to find a firm enough foothold, except in the thoroughly consolidated parts of our physical knowledge. Men like Einstein or Niels Bohr grope their way in the dark toward their conceptions of general relativity or atomic structure by another type of experience and imagination than those of the mathematician, although no doubt mathematics is an essential ingredient. Thus Hilbert's vast plans in physics never matured.

But his application of integral equations to kinetic gas theory and to the elementary theory of radiation were notable contributions. In particular, his asymptotic solution of Maxwell-Boltzmann's fundamental equation in kinetic gas theory, which is an integral equation of the second order, clearly separated the two layers of phenomenological physical laws to which the theory leads; it has been carried out in more detail by the physicists and applied to several concrete problems. In his investigations on general relativity Hilbert combined Einstein's theory of gravitation with G. Mie's program of pure field physics. For the development of the theory of general relativity at that stage, Einstein's more sober procedure, which did not couple the theory with Mie's highly speculative program, proved the more fertile. Hilbert's endeavors must be looked upon as a forerunner of a unified field theory of gravitation and electromagnetism. However, there was still much too much arbitrariness involved in Hilbert's Hamiltonian function; subsequent attempts (by Weyl, Eddington, 
Einstein himself, and others) aimed to reduce it. Hopes in the Hilbert circle ran high at that time; the dream of a universal law accounting both for the structure of the cosmos as a whole, and of all the atomic nuclei, seemed near fulfillment. But the problem of a unified field theory stands to this day as an unsolved problem; it is almost certain that a satisfactory solution will have to include the material waves (the Schrödinger-Dirac $\psi$ for the electron, and similar field quantities for the other nuclear particles) besides gravitation and electromagnetism, and that its mathematical frame will not be a simple enlargement of that of Einstein's now classical theory of gravitation.

Hilbert was not only a great scholar, but also a great teacher. Witnesses are his many pupils and assistants, whom he taught the handicraft of mathematical research by letting them share in his own work and its overflow, and then his lectures, the notes of many of which have found their way from Göttingen into public and private mathematical libraries. They covered an extremely wide range. The book he published with S. Cohn-Vossen on Anschauliche Geometrie is an outgrowth of his teaching activities. Going over the impressive list attached to his Collected Papers (vol. 3, p. 430) one is struck by the considerable number of courses on general topics like "Knowledge and Thinking," "On the Infinite," "Nature and Mathematics." His speech was fairly fluent, not as hesitant as Minkowski's, and far from monotonous. He had no difficulty in finding the pregnant words, and liked to emphasize short pivotal phrases by repeating them several times. On the whole, his lectures were a faithful reflection of his spirit; direct, intense; how could they fail to be inspiring? 